\title{
LE PROBLÈME DU POINT DE VUE DANS LE TEXTE DE THÉÂTRE
}

\author{
Alain RABATEL \\ IUFM de Lyon, ICAR, Université Lyon 2, \\ UMR CNRS 6175
}

Existe-t-il un point de vue dans les textes théâtraux ? La réponse dépend de ce qu'on entend par point de vue (désormais PDV), notion qui renvoie, dans le langage courant, à l'expression d'une opinion ou d'une perception et qui apparaît dans des contextes - argumentatif ou narratif - que les sciences du langage ont tendance à opposer. Si l'on interprète le PDV au sens d'opinion, de représentation personnelle ou doxique susceptible de faire se mouvoir les individus (Nonnon 1999) ou (de chaîne) d'argument(s), comme il en existe dans les interactions verbales argumentatives, alors la réponse est oui, massivement.

Si en revanche l'on restreint le PDV à un phénomène narratif, à l'instar des « focalisations "(Genette 1972, 1983), la réponse est nettement moins assurée : d'abord parce que la focalisation externe n'a aucun fondement linguistique (Rabatel $1997 a, b)$, ensuite parce que la focalisation zéro y apparaît très problématique, dans la mesure où le texte théâtral se caractérise par l'absence de narrateur primaire (1). En sorte que la seule instance disponible pour un PDV est le personnage. Encore faut-il préciser que nombre d'exemples de PDV du personnage relèvent de contextes narratifs à la troisième personne (Banfield 1995, Rabatel 1998), alors que les tours de parole des personnages renvoient à l'énonciation personnelle, ce qui limite énormément les PDV hétérodiégétiques, le texte de théâtre reposant rarement sur le plan d'énonciation historique, sauf dans quelques monologues narratifs. Reste donc une focalisation interne à la première personne, ce qui semble restreindre la portée du phénomène.

Ces deux réponses contrastées invitent à revenir sur la définition du PDV. On le fera à partir de sa nature radicalement polyphonique, permettant au locuteur d'exprimer son PDV, ou de se positionner face aux PDV de ses interlocuteurs ou des tiers délocutés, tels qu'il les reconstruit dans son propre discours ; en tentant de

(1) Rappelons que Genette donne trois acceptions distinctes de la notion, comme absence de focalisation (= absence de PDV du narrateur), comme PDV du narrateur, ou comme PDV omniscient résultant de la somme de toutes les autres focalisations : cf. Rabatel $1997: 62-68$. Sur le fond, on n'aura toutefois garde d'oublier que le personnage peut produire des récits seconds... 
saisir les relations entre PDV argumentatif et PDV narratif (2). Notre thèse est que le PDV, dont les énoncés en apparence les plus anodins ont, du fait de leur dialogisme, une valeur argumentative directe ou indirecte, enrichit l'analyse interactionnelle, en donnant une incarnation linguistique aux dimensions psychologique, idéologique ou sociologique des personnages construites par l'interaction même. Autrement dit, le PDV aide à l'interprétation du texte théâtral par le biais de l'enrichissement énonciatif de la dimension interactionnelle (3).

\section{CADRE THÉORIQUE}

\subsection{Point de vue et polyphonie}

Le PDV sera ici défini d'une manière suffisamment générale pour pouvoir rendre compte de la construction d'un PDV indépendamment du fait que ce PDV apparaisse en contexte narratif ou dans un autre contexte, argumentatif, informatif, etc., comme indépendamment des plans d'énonciation choisis.

On nommera PDV tout ce qui, dans la référenciation des objets (du discours) révèle, d'un point de vue cognitif, une source énonciative particulière (locuteur/énonciateur ou énonciateur, cf. infra, 1.2.) et dénote, directement ou indirectement, ses jugements sur les référents - d'où l'importance des dimensions axiologiques et affectives du PDV.

Si les points de vue sur les référents sont sensibles à travers les dimensions cognitives, axiologiques et affectives qui émergent à propos de la référenciation, alors il n'y a nulle raison, sur le plan théorique, pour limiter la problématique du PDV aux perceptions, et ce, d'autant plus que l'expression linguistique des perceptions ne sépare pas entre perceptions d'un côté, pensée et/ou éventuellement paroles de l'autre. Il est donc plus satisfaisant de considérer que le PDV est une forme générale d'expression de la subjectivité d'un sujet, telle qu'elle s'exprime à propos de la référenciation d'un objet du discours, qui peut s'accommoder tantôt de comptes rendus de perceptions, tantôt de comptes rendus de paroles, tantôt de comptes rendus de pensées.

Ainsi envisagé, le PDV est proche du discours représenté/rapporté (DR); sur le plan syntaxique, le PDV peut emprunter le rapport direct, indirect, indirect libre, direct libre, voire suivre un mode d'expression paratactique qui, en l'absence de lien hypotaxique, rend nécessaire la prise en compte de relations sémantiques entre les énoncés (4):

[1] Compte rendu direct de parole :

Pierre s'approcha de la fenêtre, regarda le convoi et dit : « l'assistance est nombreuse »

(2) Compte tenu du caractère inédit de cette confrontation de la problématique du PDV avec le texte théâtral, le choix des exemples a été guidé par des préoccupations théoriques, indépendamment du souci de rendre compte de la spécificité de tel ou tel genre ou sous genre, selon les époques.

(3) Comme Ryngaert 1999: 1, nous ne partageons pas le scéno-centrisme selon lequel les analyses littéraires ou linguistiques du texte théâtral devrait être récusées pour cause de manque originel qui ne serait comblé que par la représentation. Nos analyses énonciatives et interactionnelles relèvent plutôt du texto-centrisme, en ce qu'elles s'appuient fondamentalement sur les textes - et sur le contexte, puisque nous nous réclamons de la pragmatique -, et pas sur leurs mises en scène, quand bien même nos analyses ont des implications que les mises en scènes doivent éprouver.

(4) C'est ce qui se passerait si [3] faisait l'ellipse du verbe de perception, le lecteur devant inférer le mouvement perceptif de Pierre dans les propositions au PS, et attribuer le commentaire et la perception dans le deuxième plan à Pierre, malgré l'absence de lien hypotaxique (que les deux points ne remplacent pas totalement) ; cette absence serait plus forte si les deux points étaient remplacés par un point. Le raisonnement est identique en [10], [11] et [12]. 
[2] Compte rendu direct de pensée :

Pierre s'approcha de la fenêtre, regarda le convoi et se dit : "l'assistance est nombreuse »

[3] Compte rendu direct de perception :

Pierre s'approcha de la fenêtre et regarda le convoi : l'assistance était nombreuse/l'assistance est nombreuse (5).

[4] Compte rendu indirect de parole :

Pierre s'approcha de la fenêtre, regarda le convoi et dit que l'assistance était nombreuse

[5] Compte rendu indirect de pensée :

Pierre s'approcha de la fenêtre, regarda le convoi et pensa que l'assistance était nombreuse (6).

[6] Compte rendu indirect de perception :

Pierre s'approcha de la fenêtre, regarda le convoi et remarqua que l'assistance était nombreuse.

Pierre vit que l'assistance était nombreuse.

[7] Compte rendu indirect libre de parole :

Pierre s'approcha de la fenêtre, regarda le convoi. II attira l'attention de Jean. L'assistance était nombreuse!

[8] Compte rendu indirect libre de pensée :

Pierre s'approcha de la fenêtre, regarda le convoi. Que l'assistance était nombreuse!

[9] Compte rendu indirect libre de perception :

Pierre s'approcha de la fenêtre, regarda le convoi. L'assistance était vraiment nombreuse!

[10] Compte rendu direct libre de parole :

Pierre s'approcha de la fenêtre, regarda le convoi. Que l'assistance est nombreuse!

Cette remarque fut accompagnée d'un sourire.

[11] Compte rendu direct libre de pensée :

Pierre s'approcha de la fenêtre, regarda le convoi. L'assistance est nombreuse.

Cette pensée fugace le réconforta.

[12] Compte rendu direct libre de perception:

Pierre s'approcha de la fenêtre et regarda le convoi : assistance nombreuse, deux cents personnes environ/Pierre s'approcha de la fenêtre et regarda le convoi. Spectacle réconfortant d'une assistance nombreuse, deux cents personnes environ.

[13] Compte rendu narrativisé de parole (= discours narrativisé)

Pierre discuta une heure avec Charles

[14] Compte rendu narrativisé de pensée (= psycho-récit)

Pierre imagina les bonnes raisons que Charles alléguerait

[15] Compte rendu narrativisé de perception (= PDV embryonnaire)

Pierre s'amusa à voir un Charles hésitant avant de prendre la parole

Discuter longuement ces exemples nous détournerait inutilement de notre objectif (7). Bornons-nous à remarquer la parenté des relations hypotaxiques de [3] et des exemples [10]-[12], qui explique que les énoncé en italiques puissent légitimement être interprétés, selon les données co-textuelles (en gras), comme un compte rendu direct de perception, ou, du fait de l'absence de données contex-

(5) L'IMP rapproche conventionnellement le PDV du DIL et le présent du discours direct libre. Une telle distinction repose sur la survalorisation quasi exclusive de l'IMP dans le DIL, ce qui mérite d'être discuté.

(6) Cette possibilité est très fréquente dans les discours rapportés oraux comme l'a montré une récente analyse de Marnette $2002: 218-220$, selon laquelle, en français parlé, les pensées ne sont pas rapportées au DIL (seulement $4 \%$ des formes répertoriées dans les corpus du GARS inventoriés), mais majoritairement au DI.

(7) Pour une analyse plus détaillée des relations entre PDV et DR, cf. Rabatel 2003b, f, h. 
tuelles, comme un compte rendu direct libre de parole ou de pensée. De même, en [12], si « spectacle » oriente l'interprétation vers un compte rendu direct de perception, en revanche, " assistance nombreuse, deux cents personnes environ " dénote une perception intentionnelle, une activité cognitive pouvant s'interpréter comme compte rendu de pensée, voire comme compte rendu de parole, si l'on considère que le mouvement de la pensée est (infra)verbalisé. Cette hésitation n'affaiblit pas l'analyse, elle exhibe au contraire les continuums existant sur les plans phénoménologique et cognitif entre perception, pensée et parole (Damasio 1994, 1999), tout comme sur le plan proprement linguistique (Rabatel 2001c, 2003b). Cette parenté linguistique explique, sur le plan stylistique, l'extrême labilité des perceptions, des pensées et des paroles, et, sur le plan sémiotique, la similitude de leurs valeurs textuelles : ce n'est pas un hasard, en effet, si les valeurs textuelles de construction de la mimésis, de la mathésis et de la sémiosis (Adam et Petitjean 1989) sont semblables pour les descriptions comme pour les paroles de personnage.

Lorsque le PDV indique un compte rendu de perception développé dans le second plan, comme dans les italiques de [3], on parlera de PDV « représenté " (Rabatel 1998 : 54) ; lorsque le PDV perceptif se limite à des traces dans le premier plan (8), comme en [15], on parlera de PDV « embryonnaire » (Rabatel 2000, 2001a). Lorsqu'il est mêlé à l'expression de paroles ou de pensées, le PDV peut être dit « asserté » et s'apparente à telle ou telle forme conventionnelle de discours rapporté (Rabatel 2001c, 2003c, f, h). Si l'on repense à notre question initiale, on mesure à présent que la réponse ouvre un vaste champ de possibles : le PDV représenté se rencontre bien dans le texte théâtral, lorsque les personnages endossent une fonction narrative et sont amenés à décrire le cadre spatio-temporel, les événements, les personnages, à (se) dénommer, (se) désigner, d'une manière plus ou moins mimétique. Le PDV raconté apparaît à chaque fois que le locuteur rapporte ces mêmes objets du discours de façon diégétique, qu'ils soient antérieurs ou simultanés par rapport au présent de l'énonciation. Le PDV asserté se manifeste dans toutes les manifestations du DR, et plus particulièrement dans les énoncés embrayés du DD, que les locuteurs jugent directement, sous la forme de commentaires explicites, ou indirectement, à travers les stratégies de dénomination, indépendamment de toute chaîne explicite d'arguments hiérarchisés par des connecteurs.

Avec le PDV asserté, on sent bien que PDV ouvre à l'analyse tout le champ des énoncés à visée ou à dimension argumentatives (9). Dès lors, le PDV sort du domaine du DR, qui présente pourtant un cadre privilégié à l'expression d'un PDV : non seulement le PDV ne se cantonne plus à l'expression de perceptions, mais encore il ne se limite plus au DR. Le PDV peut exprimer des opinions ou jugements de valeur, sans que ce jugement paraisse subjectif, et indépendamment de la présence de marqueurs traditionnels de l'argumentation (arguments, connecteurs logico-argumentatifs, du type « les gens étaient pressés les uns contre les autres, par conséquent la foule était très nombreuse »), car le choix d'une dénomination, dès le cadre de prédication, telle "foule », suffit pour orienter l'énoncé dans un sens déterminé :

[16] La foule était nombreuse.

[17] Les manifestants étaient vraiment nombreux.

(8) Ce qui le rend proche du psycho-récit ou du discours narrativisé.

(9) Amossy 2000 distingue ainsi les énoncés à visée argumentative directe avec des (chaînes d') arguments explicites, des connecteurs, activant une forte activité déductive, tandis que les énoncés à dimension argumentative (indirecte) orientent les interprétations sans passer par les marques traditionnelles de l'argumentation (logos) et reposent sur des stratégies lexicales, stylistiques, entraînant des calculs inférentiels de nature plus ou moins implicite, privilégiant davantage le pathos et l'ethos. 
II serait simpliste de croire que le PDV se limiterait à un modus subjectif auquel s'opposerait un dictum objectif : c'est d'emblée au niveau du dictum, à travers notamment la sélection, la catégorisation ou encore la structuration qu'opèrent les modalités, comme, en [18], le choix de " populace » et le mode indicatif qui indique un point de vue sous la forme d'un fait objectif, donc non sujet à discussion. Quant à la modalisation, elle concerne la distance du locuteur envers son dire, à travers les dédoublements énonciatifs, les commentaires réflexifs, etc. : cf. « vraiment ", "sans aucune gêne ». Aussi chacun des énoncés précédents peut-il comprendre plus ou moins de subjectivèmes, qui donnent un tour plus ou moins subjectivant au PDV (10).

II faut insister sur le fait que les énoncés ne mentionnent pas toujours clairement l'origine énonciative du PDV : les marques ouvrantes et fermantes sont certes aisément repérables dans le DD, moins dans le DI, parce que si on sait où commence le DI, on ne sait pas toujours clairement où il s'arrête et où commence le PDV du locuteur citant. Les bornes ouvrante et fermante sont moins nettes encore dans le DIL, le DDL, le discours narrativisé ou dans le PDV représenté. Semblablement, les marqueurs de portée (Charolles 1988), tels " d'après », " selon », etc. indiquent bien l'ouverture d'un espace énonciatif autre dans le discours du locuteur, sans toujours préciser où s'arrête ce même PDV. Et le problème est encore plus complexe lorsque le PDV se limite à l'inclusion de subjectivèmes en l'absence de bornes ouvrante et fermante. Ainsi, dans les exemples [1]-[15], en contexte de DR, le changement de tiroir verbal, notamment de visée, joue un rôle de bornage énonciatif en indiquant un débrayage énonciatif ténu (Rabatel 2003b).

Le PDV est donc une problématique transversale, qu'on retrouve dans les récits comme dans les argumentations, invitant ainsi à analyser les passerelles entre ces deux pôles fondamentaux de l'activité langagière (Rabatel 2000 et 2004).

Dans ces conditions, la réponse précédente à notre question initiale se voit complétée par le fait que, comme le texte théâtral met aux prises des personnages en conflit, dans des situations rituelles ou ordinaires, ils argumentent directement ou indirectement, en sorte que le PDV se rencontre au théâtre derrière toutes les formes directes ou indirectes d'argumentation.

\subsection{Les instances du point de vue}

Selon la distinction opérée par Ducrot $1984: 204$, on distinguera locuteur et énonciateur (11), qui entretiennent des relations pouvant être formalisées comme suit :

[1] INSTANCES : Le locuteur (L) (12) est l'instance qui profère un énoncé, selon un repérage déictique ou anaphorique. L'énonciateur $(E)$, proche du sujet modal de Bally, assume l'énoncé. Dans un énoncé monologique, le locuteur est aussi énonciateur ; on notera par une majuscule, suivie du chiffre 1, ce locuteur primaire et cet énonciateur primaire, et par une barre oblique le syncrétisme de L1 et de E1. Dans un énoncé dialogique combinant deux PDV, le deuxième n'est pas nécessairement exprimé par un 12 cité, mais par un e2 : ainsi des « discours " narrativisés, des « discours » indirect libre à la troisième personne, des mentions écho ironiques, ou des PDV re-

(10) Cf. Rabatel $2003 \mathrm{~h}$, pour une exemplification de gradients de mimétisme.

(11) Charaudeau et Maingueneau : 2002, 220-224, 226.

(12) Sans compter le sujet parlant, ou producteur empirique de l'énoncé, dont la définition ne fait pas débat, par rapport au locuteur et à l'énonciateur. 
présentés ou embryonnaires en contexte hétérodiégétique, toutes formes qui relèvent à un titre ou à un autre d'un certain effacement énonciatif (Vion 2001, Rabatel 2003e).

[2] STRUCTURES : Dans un cadre dialogal ou dialogique, on notera respectivement, par une minuscule suivie du chiffre 2, 12 et e2 les locuteurs et énonciateurs enchâssés (ou cités) dans l'énoncé du locuteur citant, et dans le point de vue originel à partir duquel se marquent les positions énonciatives divergentes. En ce sens, $L$ et $E$ sont :

- linguistiquement premiers, par rapport à l et à e qui occupent une posture seconde, puisque la deixis est calculée par rapport à $L 1$, impliquant les transformations idoines dans le discours cité de 12 ;

- hiérarchiquement supérieurs à I et à e, sur le plan pragmatique, dans la mesure où $L 1$ rend compte des PDV de 12 en fonction de ses propres intérêts de locuteur primaire.

[3] LIENS SEMANTIQUES : les relations entre L1/E1 et I2/e2 relèvent :

- tantôt de la responsabilité ou de la non responsabilité, lorsque les relations sont explicites;

- tantôt de la consonance ou de la dissonance (Cohn 1981), lorsque ces liens sont implicites. Dans les deux cas, ces liens sont graduels.

Ainsi, les italiques de [3] correspondent au PDV de e2, Pierre : le discordanciel énonciatif entre premier et second plan, le choix d'une forme de visée sécante, le rapport sémantique entre la perception dans le premier plan (fait, perception appréhendée comme un tout) et dans le deuxième plan (commentaire, perception appréhendée dans ses parties) renvoie à la perception de Pierre, malgré l'absence de marquage hypotaxique. Ce PDV représenté, qui pourrait aisément se développer dans un fragment descriptif, est différent du PDV embryonnaire de [15] où Pierre, exprime son PDV sur Charles dans un premier plan, sans développer de commentaires dans un deuxième plan. Dans les deux cas, le PDV est celui du sujet saillant, Pierre, comme dans tous les exemples précédents ; à défaut, le PDV serait celui du narrateur ; mais, dans le contexte, L1, qui rapporte ce PDV, fait entendre au destinataire que le PDV est fiable, en l'absence de marque de distance explicite.

Ce modèle théorique est particulièrement rentable pour le texte narratif, pris en charge par un locuteur narrateur (qu'il faut théoriquement disjoindre du sujet parlant). Quelle est sa pertinence pour le texte théâtral, caractérisé par l'absence de narrateur? Ubersfeld remarque à juste titre que

Si E1 (13) construit le discours qu'assume le personnage E2 et dont celui-ci s'affirme auteur et responsable, le scripteur E1 dégage sa responsabilité de ce discours ; et il est vain de le lui attribuer. Non, Alceste, n'est pas le porte-parole de Molière, pas plus que Philinte, d'ailleurs. II n'y a pas d'auteur-scripteur dont la voix se fasse entendre directement dans le dialogue de théâtre ; l'auteur E1 est responsable de la totalité du texte de théâtre (fable + paroles de tous les personnages + didascalies), non de la parole de chacun - tandis que l'énonciateur-personnage E2 est « responsable » de la totalité de ses répliques (Ubersfeld 1996 :11).

Cela pose le problème de savoir comment repérer des segments textuels référant, sinon explicitement, du moins implicitement au PDV du scripteur. L1 (14), dans le cadre du texte théâtral écrit, n'est pour sa part responsable que du titre,

(13) Ubersfeld, comme Kerbrat-Orecchioni, utilisent E là où nous parlons de locuteur. Cf. infra.

(14) L1, auteur du drame, n'est pas un locuteur-narrateur, mais un locuteur-dramaturge. 
des avant-textes éventuels (préface, avis au lecteur, avant-propos, dédicace, etc.), de la liste des personnages assortie de leurs relations et des didascalies. Certes, L1 est aussi l'auteur des propos des I2, et il peut, sur le plan des liens sémantiques, être plus ou moins proche de tel ou tel, mais: en l'absence de trame narrative, de verbes du discours attributifs, de descriptions ou de commentaires du narrateur (Lintvelt 1981), il n'y a pas de lien explicite de (non)responsabilité (sauf ceux qui figurent dans les péri-textes), et l'on se trouve face à des liens de consonance ou de dissonance, nettement plus complexes, sur le plan interprétatif. C'est là une des difficultés supplémentaires du texte de théâtre sur le texte narratif, la nature particulièrement " lacunaire » (ou paresseuse) du texte théâtral obligeant le lecteur (15) à un surcroît de travail interprétatif.

Maingueneau (après Isacharoff 1985) utilise le concept d'archi-énonciateur pour rendre compte de ce fait. L'archi-énonciateur serait une instance (distincte de celle de l'écrivain) subsumant toutes les positions énonciatives et prenant en charge un réseau conflictuel de positions énonciatives (Maingueneau $1990: 141-2$ ). Même si l'archi-énonciateur n'est pas l'écrivain, on sent bien qu'il est proche malgré tout d'une instance textuelle énonciative supérieure, quelque chose comme l'écrivain textualisé. Cela souligne la complexité de la question herméneutique et de son substrat énonciatif, sans régler le problème de la nature des relations énonciatives de consonance ou de dissonance entre le locuteur-dramaturge (L1) et ses personnages (I2), ce que nous tenterons de préciser à travers les concepts de coénonciation, de sur-énonciation ou de sous-énonciation, en revisitant le concept de double énonciation.

\subsection{Point de vue et double énonciation}

Ces données complexifient le cadre communicationnel du texte théâtral présenté comme un phénomène de "double-énonciation " (Kerbrat-Orecchioni 1984). Rappelons que cette double énonciation dédoublée oppose d'un côté deux émetteurs, le locuteur-dramaturge $\mathrm{L} 1$ et les personnages 12 , avec, deux récepteurs distincts : I'allocutaire de L1 est le spectateur (16) ou le lecteur, tandis que les allocutaires de 12 sont les personnages - le plus souvent sous la forme d'un allocutaire destinataire direct, mais aussi d'un destinataire indirect ou encore d'un récepteur additionnel dont la présence échappe totalement à la conscience du locuteur. La double énonciation signifie que la communication ne se limite pas aux interactions entre les 12 , mais intègre le fait que le discours du scripteur (c'est-àdire la somme des interactions des I2) s'adresse par-dessus les I2, au spectateur/lecteur. Si l'on intègre la dimension scénique, les pôles émetteur et récepteur ne sont plus doubles, mais triples : d'un côté, le scripteur + le personnage + l'acteur, de l'autre le personnage + l'acteur + le spectateur (Ubersfeld $1996: 9-10)$. Si l'on prend en compte la montée en puissance du metteur en scène, il faut rajouter une quatrième instance dans ce schéma, avec le metteur en scène, d'un côté, et, de l'autre, un destinataire qui appartiendrait au champ de la critique théâtrale.

Contrairement à une idée reçue la double-énonciation n'est pas propre au théâtre, quand bien même ce dernier institutionnalise et par là-même objectivise ce rapport communicationnel démultiplié. On le retrouve aussi avec le texte narratif, car le narrateur (et aussi, dans une mesure variable, l'écrivain) s'adresse à un lecteur modèle, ou à des lecteurs réels, par-dessus les interactions entre per-

(15) Ce surcroît est moindre si des médiations aident à combler les vides : éditions scolaires des œuvres, ou mises en scène. Il en découle qu'en général, on comprend plus facilement un texte grâce à sa représentation que par sa seule lecture cf. Ryngaert 2000 et Petitjean 2004.

(16) Ce spectateur, comme Ubersfeld le rappelle, se scinde en « un destinataire imaginaire D2' et un réel D2, avec toutes les béances possibles entre les deux » (Ubersfeld 1996 : 11). 
sonnages, qui peuvent être incarnés à l'écran par des acteurs. Au surplus, l'expression "double-énonciation » renvoie à une acception de l'énonciateur qui en fait l'équivalent du locuteur. Ubersfeld reproduit ce schéma en parlant de E1 (le scripteur) et de E2 (le personnage) là où nous parlons de L1 et de I2. II est certain que dans les énoncés monologiques, il y a bien syncrétisme entre L1 et E1, entre 12 et e2, mais on sait que ces énoncés monologiques sont finalement assez rares, sauf sur de courtes portions d'énoncés, et que, pour rendre compte des énoncés dialogiques, on a besoin de distinguer entre locuteur et énonciateur, dès lors que l'énoncé comporte des PDV différents, sans que ces derniers ne renvoient tous à des locuteurs enchâssés spécifiques. II n'y a pas d'intérêt à plaquer l'énonciateur sur le locuteur, dans la mesure où cela laisse penser que toute co-énonciation repose sur de la co-locution. Or le théâtre est précisément un genre qui institutionnalise cette déliaison des instances, car il n'y a pas co-locution entre le scripteur - ou le metteur en scène - et le lecteur - ou le public - (et réciproquement) (17) : il y a certes de la " communication » entre eux, par-dessus la tête des personnages, mais elle repose sur un phénomène de sur-ou de sous-énonciation sans co-locution (cf. infra) : car en bonne logique, un allocutaire est une instance en capacité puissantielle de parole responsive, adressée à $L 1$, en situation de coprésence ou en situation de communication à distance (spatiale ou temporelle). Or, si cette qualité est bien le propre des allocutaires de 12, ce n'est pas le cas général des allocutaires de L1 : certes, le public peut réagir, le lecteur s'indigner ou s'enthousiasmer, il parle alors à propos de, mais il ne parle plus à L1, qui, souvent, n'est plus là depuis belle lurette pour lui répondre (18). Cette situation inégale est décisive pour l'herméneutique du texte théâtral ; elle joue davantage pour l'auteur que pour le metteur en scène, qui, lui, peut ajuster selon les réactions du public : en ce sens, les relations metteur en scène et spectateur ne se contentent pas de redoubler celles entre auteur et lecteur.

Enfin, il convient de distinguer entre lecteur et spectateur, car, ces deux co-énonciataires ne sont pas toujours dans la même situation cognitive par rapport à l'œuvre. Une didascalie initiale extradiégétique, de nature proleptique, apporte des indications pour le lecteur (et le metteur en scène) mais pas pour le spectateur, et n'a donc pas le même statut que les didascalies intradiégétiques qui présentent au spectateur un apport d'informations au fil du texte. C'est ce que montre fort bien la didascalie extradiégétique de L'Affaire Ducreux, qui comprend in fine une indication proleptique dont le spectateur n'aura connaissance que dans les derniers moments de la mise en scène :

[19] Une femme dans la soixantaine, ancienne domestique.

Elle est d'abord assise à la table de sa cuisine, une lettre devant elle, qu'elle consulte à intervalles irréguliers.

La lettre est pleine de questions qu'elle lit soit sans voix, soit à voix basse et auxquelles elle improvise des réponses à haute voix.

C'est une femme qui paraît maîtresse d'elle-même au début de la scène. Mais on s'aperçoit progressivement qu'elle ne domine pas sa nervosité et qu'elle perd la tête. [...]

Il y aura en fin du monologue une voix off, impérative, qui remplace les questions lues et à laquelle elle répond de façons incohérente. [...]

A la fin de la scène la caméra se dirige vers la table et se fixe sur la lettre. On constate que ce n'est qu'une feuille blanche, sans rien d'écrit (Pinget L'Affaire Ducreux, Minuit, $1995: 9$ ).

(17) Le cas est un peu différent pour la relation acteur/public, lorsque le personnage s'adresse directement au public. Mais cette communication reste très inégale, le public n'est pas censé répondre par des actes de discours concernant la diégèse ; qui plus est, cette relation très inégalitaire n'existe que lorsque le texte est mis en scène, elle ne se manifeste pas lorsque le texte est lu.

(18) Sauf dans les cas où la critique génétique dispose d'échanges entre l'auteur et certains de ses lecteurs privilégiés, autour du texte en devenir. 
Ainsi, à la différence du lecteur, c'est seulement au tomber de rideau que le spectateur apprend que la feuille est une feuille blanche. Cette situation montre la profonde dissymétrie dans la double énonciation, entre lecteur d'un côté et spectateur de l'autre. Il en est bien d'autres.

\subsection{La prise en charge énonciative des points de vue : faits de co-énonciation, sous-énonciation et sur-énonciation dans la double énonciation}

Comme on l'a dit, le concept d'archi-énonciateur, trop en relation avec l'instance écrivain, n'offre pas assez de jeu pour rendre compte des phénomènes de prise en charge énonciative entre locuteurs et entre énonciateurs eux-mêmes. Les instances parties prenantes de la « double énonciation » sont toutes susceptibles d'entretenir des relations relevant de la co-, sur- et sous-énonciation. De quoi s'agit-il ? Un PDV peut être dominant sans pour autant que le locuteur parle beaucoup, comme c'est le cas pour les autorités dont la parole est rare, et dont le PDV est exprimé par ceux qui sont sous sa coupe, sans que, nécessairement, ils le partagent : dans ce cas, le PDV interactionnellement dominant renvoie à celui d'un sur-énonciateur (Rabatel 2003a, c, d), et le PDV interactionnellement dominé à un sous-énonciateur (Rabatel 2002, 2003e) ; quant aux co-énonciateurs, ils sont interactionnellement co-producteurs d'un PDV véritablement commun, partagé - ce faisant, notre conception de la co-énonciation diffère de celle de Jeanneret 1999 dont les exemples de co-énonciation se réduisent pour la plupart à de la colocution (Rabatel 2003c).

1.4.1. Relations entre personnages : nous nous limiterons ici à une rapide exemplification de ces postures.

\subsubsection{Co-énonciation :}

[20] Rodrigue: O miracle d'amour!

Chimène : O comble de misères!

Rodrigue: Que de maux et de pleurs nous coûteront nos pères !

Chimène: Rodrigue, qui l'eût cru ?

Rodrigue: Chimène, qui l'eût dit ?

Chimène: Que notre heur fût si proche et sitôt se perdît?

Rodrigue: Et que si près du port, contre toute espérance,

Un orage si prompt brisât notre espérance?

Chimène: Ah! mortelles douleurs!

Rodrigue: $\quad$ Ah ! regrets superflus ! (Corneille, Le Cid, III, 4).

Cette célèbre stichomythie correspond à la co-énonciation d'un PDV commun : certains tours de paroles réunis des deux amants forment une intervention (qu'il faut distinguer des répétitions syntaxiques caractéristiques du chant amoebée), comme lorsque Chimène et Rodrigue évoquent leur bonheur si proche, avec le rôle des subordonnants en tête de vers. Dans le co(n)-texte, cet énoncé commun construit bien une référence partagée, qui est la marque même de l'unisson amoureux. Mais il n'en va pas toujours ainsi, et l'on pourrait aisément opposer des situations amoureuses où l'on voit un amoureux co-produire un énoncé amorcé par l'amante dans le sens attendu par la belle, sans être pour autant sincère : c'est la figure bien connue du séducteur, qui devine à l'avance les mots qu'il faut dire, et sait le ton qu'il faut employer: malgré son apparence monologique, l'énoncé est en réalité dialogique, derrière l'apparence d'accord et de fusion, et la situation dramatique repose sur cette discordance énonciative et épistémique, l'amante se croyant aimée cependant que le séducteur, comme le public, sait qu'il n'en est rien. 
1.4.1.2. Sous-énonciation : c'est la posture de Dom Juan, lorsqu'il s'adresse à Dom Louis en disant regretter sa conduite passée

[21] Oui, vous me voyez revenu de toutes mes erreurs; je ne suis plus le même d'hier au soir, et le ciel, tout d'un coup, a fait en moi un changement qui va surprendre tout le monde (Molière, Dom Juan $\mathrm{V}, 1$ ).

Il ne pense pas ce qu'il dit, et adopte une posture de sous-énonciateur, en référence à l'image dominante (Noblesse oblige) dont se réclame Dom Louis, et par rapport à laquelle il se situe, en multipliant les reformulations de la contrition, incarnées à travers un ton, des gestes, des taxèmes idoines, ainsi qu'y invite la didascalie enjoignant l'acteur à faire "l'hypocrite ». De même, lorsque Sganarelle en habit de médecin se sent de l'humeur à disputer sur l'existence de Dieu, il a beau parler beaucoup, en tant que locuteur, il sent bien qu'il a de la peine à dérouler un raisonnement convaincant, manifestant par ses hésitations, ses faiblesses de raisonnement, ses mimiques et sa gestuelle, sa posture de sous-énonciateur:

[22] Oh ! dame, interrompez-moi donc, si vous voulez. Je ne saurais disputer si l'on ne m'interrompt. Vous vous taisez exprès, et me laissez parler par belle malice (Molière, Dom Juan, III, 1).

En [21], le sous-énonciateur renvoie une posture dominée choisie par tactique (Dom Juan empruntant ce masque pour continuer sur sa logique en trompant son entourage), tandis qu'en [22], Sganarelle est sous-énonciateur parce qu'il n'en peut mais, face à son maître, ce qui ne l'empêche pas, avec d'autres personnages, d'adopter la posture dominante qui est habituellement celle de son maître.

1.4.1.3. Sur-énonciation: dans les deux premières scènes d'exposition du Tartuffe, le personnage éponyme, sans dire un mot (il ne sera présent qu'à l'acte III), est au centre de tous les échanges, notamment dans la scène 2 de l'acte I, Tartuffe étant présent dans les réactions d'Orgon, comme dans l'évocation contrastée des malaises d'Elmire et des aises du soi-disant « pauvre homme ». Mais le sur-énonciateur n'est pas nécessairement celui dont la parole est rare : dans la scène 4 de l'acte II de Dom Juan, ce dernier occupe une position haute, entre Charlotte et Mathurine, avec Sganarelle comme public : ce sont ses interlocutrices qui le mettent en position centrale, se positionnent en demandeuses, comme le signalent leurs actes de langage.

Compte tenu de la rentabilité de ces postures pour l'analyse des interactions entre personnages, il paraît utile d'insister (sur un mode plus prospectif que conclusif) sur certaines implications :

- Ces postures de co-, sur- et sous-énonciation entre personnages ne doivent pas être réifiées, ontologisées, rien ne serait plus faux que d'assimiler une posture énonciative déterminée par des rapports de force momentanés entre locuteurs à des traits psychologiques ou sociologiques permanents. Ainsi, nombre de scènes mettant en relation maîtres et valets, avec un valet souvent doté d'infirmités discursives et en situation de sous-énonciation par rapport au maître voient à certains moments les rapports s'inverser. En effet, de personnage à personnage (comme dans la vie), les rapports de co-, sur- et sous-énonciation sont surdéterminés par la dialogie interactionnelle et par le fait que dans le procès dialogique même se rejouent (19) constamment des problèmes de place, de rôle, de stratégie qui sont certes déterminés par la situation objective antérieure, mais qui doivent être vérifiés (ou contestés) au cours même de l'interaction : les relations ver-

(19) Nous tenons beaucoup au préfixe re-, qui a certes une valeur intensive, mais qui indique surtout que les échanges sont déterminés en amont par la situation : l'oublier, serait verser dans l'idéalisme ; mais, comme on vient de le dire, survaloriser la situation reviendrait à verser dans les errements des théories du reflet, qui avaient cours dans les années 1960 et 1970. 
ticale (statut, place) et horizontale (familiarité, distance) les relations affective (attraction, répulsion) et idéologiques (consensus, dissensus) se nouent et dénouent sans cesse dans l'interaction, à travers échanges symétriques ou dissymétriques, à la manière des interactions entre ethos préalable, ethos pré-discursif et ethos discursif (Maingueneau 1984, 1987). Qui plus est, le théâtre offre maintes situations dans lesquelles le personnage est habité par un rôle ou par un statut (20), et se met à parler en maître, en aristocrate, ou en valet (cf. infra, en 3., les exemples empruntés à Molière et à Marivaux), en sorte que « le " personnage est traversé par des rôles, des intérêts contradictoires qui rejaillissent tant sur la forme que sur le contenu des échanges et notamment des PDV. Bref, les postures énonciatives doivent absolument être disjointes de la question de la vérité et du mensonge, ou, à mieux dire, invitent à l'appréhender autrement...

— L'évocation de l'ethos ne doit rien au hasard, la manière dont les I2 « parlent leur parole » étant riche d'enseignements sur la nature du personnage, mais encore sur ses intentions pragmatiques : l'ethos partage avec le PDV le fait d'être ambivalent, d'être une forme d'intériorité sous la détermination des effets à produire à l'extérieur ( « une image de soi que le locuteur projette dans son discours afin d'emporter l'adhésion de l'auditoire », Meizoz 2003 : 243) tout en étant déterminée par ailleurs par la psychologie, la sociologie des locuteurs, ainsi que par le champ ("la scène englobante " et par la " scène générique " (Maingueneau 1987, Meizoz 2003 et Rabatel 2004). En paraphrasant Comte-Sponville (1988 : 304-305), on dira que le PDV comme l'ethos sont soumis à la double contrainte $d u$ primat du champ (social et culturel) et de la primauté des calculs pragmatico-discursifs du sujet, en sorte que cette double détermination, qui rend compte des déterminations en dernière instance du social et de la liberté (fût-elle conditionnée) du sujet, permet de dépasser l'opposition interne/externe (Meizoz 2003 : 244).

- Il découle également de ce qui précède que, si tout se rejoue dans l'interaction, alors il faut tenir compte de la structure des échanges, et distinguer dilogue et polylogue - sans oublier, bien sûr, le cas des monologues - ; nous y reviendrons dans une publication ultérieure, ou renvoyons à nos analyses d'interactions orales ou écrites en contexte didactique (Rabatel 2002, 2003a, c, d). Insistons toutefois sur le fait que si ces postures se retrouvent dans les dilogues comme dans les polylogues, elles s'objectivent plus ou moins bien selon le nombre de participants : la co-énonciation se rencontre dans les dilogues consensuels (conversation), ou dans des dilogues très codés, comme les débats entre spécialistes, dans lesquels il est habile de montrer qu'on connaît bien la pensée de l'interlocuteur, pour mieux fonder sa réfutation... ; elle est plus aisément observable dans les trilogues, qui se caractérisent souvent par des alliances à géométrie variable (deux contre un) qui créent des conditions favorables à l'émergence d'une co-énonciation contre un tiers. La sous-énonciation est plus facilement lisible dans les dilogues ; en revanche, la dimension interactionnelle de la sur-énonciation n'est jamais plus patente que dans les polylogues où l'on voit la plupart, sinon la totalité des interlocuteurs se situer par rapport à la pensée dominante du sur-énonciateur. Dans tous les cas de figure, mais plus particulièrement en contexte polylogal, les jeux énonciatifs gagnent à être croisés avec la dynamique interactionnelle, autour des modes d'imposition des thèmes conversationnels, des passations ou captations de la parole.

1.4.2. Topique énonciative et herméneutique. Cette topique énonciative n'est pas seulement utile pour l'analyse interactionnelle des personnages : I'herméneutique gagne à intégrer cette problématique, puisque toute une part de la communication

(20) Cf. les rôles (et non pas les personnages) de fou, de bouffon, ou d'enfant, à l'instar de Victor, qui chez Vitrac, profite de son statut d'enfant pour dire la vérité aux adultes (Victor). 
passe par des instances énonciatives qui construisent des interprétations (lecture ou mise en scène) par-dessus les échanges des personnages : il ne s'agit plus, comme précédemment, de parler (avec, sous, ou sur), mais de penser (avec, sous, ou sur), avant que cette pensée ne se concrétise dans un autre domaine par des paroles et ou par des actes, dans le domaine de l'art comme dans la vie quotidienne.

1.4.2.1. Relations $L 1$ (ou metteur en scène) et $I 2$ : c'est seulement en prenant l'ensemble des énoncés des 12 que L1 est archi-énonciateur. En règle générale, en considérant l'œuvre comme produit fini, L1 est sur-énonciateur par rapport aux 12: c'est là la norme, dans la mesure où le dit des personnages correspond au vouloir dire de L1 ou du metteur en scène. Cette « règle » laisse entendre qu'il y a des exceptions, rares, du côté de L1, mais toujours possibles, lorsque certains personnages et/ou la pièce se trouvent avoir auprès du public un sens différent de celui qu'y voyait son créateur, à l'instar de Beaumarchais, qui ne voyait pas dans $L e$ Mariage de Figaro une charge critique au contenu aussi radical que ce que le public jugea dès les premières représentations, et plus encore au fur et à mesure de la marche des événements révolutionnaires (21). Cette situation, comme celle de co-énonciation, se rencontre si l'on prend en compte le processus de production de l'œuvre, ainsi que le fait la critique génétique : il n'est pas impossible en effet que le processus de création de l'œuvre donne place à des faits de co-énonciation si L1, en accord avec certains de ses lecteurs, modifie son texte dans le sens souhaité par eux ; de même, les mises en scènes rituelles propres à la théâtralité rituelle analysée par Schaeffer 1999: 271 présentent, lorsque le spectateur entre dans le jeu, un autre cas de co-énonciation. Enfin, il peut y avoir sous-énonciation de $L 1$, lorsque le personnage se meut selon sa logique propre, amenant $L 1$ à modifier ses plans initiaux, voire à réécrire des pans entiers de son texte concernant tel ou tel personnage.

1.4.2.2. Relations $L 1$ et metteur en scène : l'œuvre à mettre en scène, l'éventuel péri-texte auctorial plaident en faveur d'une sur-énonciation de L1; mais L1 peut être en posture de sous-énonciateur, s'il écrit sous la commande, sans avoir de marge de manœuvre pour ses productions : ainsi de Jean Rotrou, dans les années 1630, "poète à gages " de la troupe de l'Hôtel de Bourgogne (Ryngaert 1999 : 22), ou des scénaristes aux ordres des producteurs de tout poil, de nos jours. On pourrait penser que lorsque $\mathrm{L} 1$ est son propre metteur en scène, il y a co-énonciation, mais cela n'est pas automatique. Les choses sont tout aussi variables du côté du metteur en scène, dont la marge de manœuvre fluctue selon les époques, la logique interne du champ théâtral ou la présence et la qualité des données péritextuelles de l'auteur. Selon la relation du metteur en scène envers le scripteur, il se situe dans une posture

— de co-énonciation, lorsqu'il se positionne en exégète respectueux de l'œuvre, cherchant à viser une vérité du texte à travers une lecture actuelle de l'œuvre ; - de sous-énonciation lorsqu'il respecte servilement la doxa relative aux intentions de l'auteur et/ou aux mises en scènes antérieures se position en interprète fidèle, en serviteur de l'œuvre ;

- de sur-énonciation, lorsque le metteur en scène fait abstraction du contexte de production et notamment du péritexte auctorial, se situant dans une posture distanciée d'analyste de l'œuvre (voire de l'auteur), produisant des interprétations qui contreviennent explicitement à certaines analyses de l'auteur, ou à la tradition.

(21) Ces changements dans la réception des œuvres sont une des manifestations des phénomènes de sur- ou de sous-énonciation. 
Ces attitudes de sous-, co- ou sur-énonciation, qui témoignent de la posture énonciative-interprétative du metteur en scène, n'impliquent pas nécessairement que la seule attitude éthiquement ou esthétiquement juste soit la co-énonciation : certaines adaptations fidèles peuvent être dénuées de pertinence, tellement le message est aseptisé, tandis que des adaptations a priori très éloignées du projet originel du scripteur sont susceptibles de déployer des aspects inédits de l'œuvre, qui s'y trouvaient potentiellement, mais auxquels on n'avait pas été attentif jusque là. Bref, le choix d'une posture énonciative n'est en rien garant de la réussite esthétique, pas plus que de la pertinence interprétative.

1.4.2.3. Le lecteur/spectateur est lui-même susceptible d'endosser des positions variées de co-énonciation, de sur-énonciation ou de sous-énonciation, tantôt envers les personnages, tantôt envers L1 (ou envers le metteur en scène) même si, en général, son statut extradiégétique lui confère une supériorité qui s'accommode fort bien d'une posture d'archi-co-énonciataire. Il est particulièrement tentant d'établir, sur le plan herméneutique, un parallélisme avec les postures de lecteur analysées par Jouve $(1992,1993)$, le lu correspondant à une sorte de sous-énonciateur, le lisant à une sorte de co-énonciateur, et le lectant jouant ou interprétant à une sorte de sur-énonciateur.

\section{LE PARADOXE PRAGMATIQUE DU POINT DE VUE DANS LE TEXTE THÉÂTRAL : PETITES MANIFESTATIONS, GRANDS EFFETS}

II résulte de la nature polyphonique du langage que les personnages peuvent reproduire, contrefaire, imaginer, déformer les paroles et les pensées d'interlocuteurs ou de tiers. Les verbes par lesquels le narrateur accède aux pensées ou aux paroles ne lui sont pas réservés. Voilà pourquoi les discussions sur l'impossibilité qu'un personnage en focalisation interne accède aux pensées d'un autre personnage (Genette, Bal $1977: 42$, Lintvelt 1981, Vitoux 1982 : 363, cf. Rabatel 1997) n'ont aucun fondement énonciatif, et ne peuvent à la limite être interprétées qu'en termes de fiducie. L'intérêt de l'analyse de la parole des personnages réside ainsi dans le fait qu'ils expriment leur PDV, mais aussi se positionnent par rapport aux PDV des autres, qui peuvent être

- soit rapportés sous la forme de discours de 12 cités,

— soit représentés dans des énoncés délocutés coréférant à des e2 - DIL à la troisième personne, discours narrativisés, psycho-récit, PDV représentés, PDV racontés/embryonnaires, mentions écho ironiques, etc.

On limitera l'examen à quelques cas de polyphonie dont l'efficacité pragmatique est inversement proportionnelle à la discrétion de leur marquage, caractérisé par un certain effacement énonciatif.

\subsection{PDV : dénomination et désignation dans les dialogues}

Comme on l'a dit à propos des exemples [16] à [18], point n'est besoin d'exprimer des arguments et d'utiliser force connecteurs pour indiquer un PDV. Les changements dans la chaîne référentielle qui caractérise Polyeucte en disent plus qu'un long discours sur les changement de PDV de Stratonice à l'égard de l'époux de sa maîtresse :

[23] Stratonice : Ce courage si grand, cette âme si divine,

N'est plus digne du jour, ni digne de Pauline.

Ce n'est plus cet époux si charmant à vos yeux ; 
C'est l'ennemi commun de l'Etat et des dieux, Un méchant, un infâme, un rebelle, un perfide, Un traître, un scélérat, un lâche, un parricide, Une peste exécrable à tous les gens de bien, Un sacrilège impie : en un mot, un chrétien.

Pauline: Ce mot aurait suffi sans ce torrent d'injures.

Stratonice : Ces titres aux chrétiens, sont-ce des impostures?

Pauline : /l est ce que tu dis, s'il embrasse leur foi ;

Mais il est mon époux, et tu parles à moi.

(Corneille, Polyeucte, III, 2, v. 776-788)

Stratonice évoque Polyeucte à travers deux chaînes anaphoriques antagonistes: les italiques renvoient au Polyeucte d'avant la conversion (les SN démonstratifs y expriment une valeur méliorative, emphatique), tandis que les anaphores définies et indéfinies en caractères gras, expriment un jugement péjoratif sur le chrétien, avec une extensité maximale pour le SN défini (comme c'est sa fonction), mais aussi pour les SN indéfinis (" un méchant» = « le méchant par excellence »), par le biais des prédications existentielles (Wilmet $1998: 142$ ).

Ce jugement ambivalent est repris sous une forme différente par Pauline, qui, en tant que romaine fidèle à ses anciens dieux, partage l'indignation de Stratonice, mais qui, en tant qu'épouse, ne peut condamner si fortement son époux: d'où le fait que le substitut qui renvoie à Polyeucte neutralise l'opposition : la première occurrence du pronom personnel dans la proposition principale, partage le jugement négatif de Stratonice sur Polyeucte converti, et la seconde, dans la proposition hypothétique, soumet ce jugement à la vérification de l'hypothèse : et le fait que cette conversion soit envisagée sur le mode hypothétique, rejaillit sur le caractère hypothétique de la condamnation, alors que le vers suivant réaffirme sans condition le caractère sacré de l'époux, tout en utilisant, ici encore, le même substitut : toutefois, l'expression marquée " il est mon époux " (vs "c'est mon époux ») témoigne du caractère instable du PDV de Pauline, le pronom personnel installant une subtile distance qui vient rompre les liens matrimoniaux : ce n'est plus cet époux fameux, c'est toujours son mari, mais un conjoint dont le comportement met en péril les liens du mariage et les sentiments amoureux. On ne saurait mieux montrer le caractère très prégnant du PDV, tel qu'il s'exprime dans la dénomination et la désignation des référents, notamment des personnages. Il faut insister sur le caractère économique de ces jugements de valeurs, qui sont certes explicites, mais qui restent très embryonnaires, et qui obéissent à des stratégies différentes: Stratonice utilise dénominations et désignations, tandis que Pauline, qui est sur la défensive, se limite à des désignations dont le flou lui évite d'avoir à nommer, c'est-à-dire à devoir prendre explicitement partie.

Ces stratégies, ici très condensées, peuvent néanmoins jouer sur de larges portions textuelles, comme on le voit dans Lorenzaccio, où les diverses dénominations du héros éponyme, disséminées tout au long de l'œuvre, indiquent d'un mot le PDV de tel personnage sur Lorenzo de Médicis. Pour ses adversaires, Lorenzo est « Lorenzaccio », voire " un Lorenzaccio » (II, 6), ou, « Renzinaccio » (IV, 7). Cet appellatif cumule une aphérèse indiquant une connivence voire une certaine familiarité et un suffixe à la valeur dépréciative exprimant un mépris certain. Pour ses amis, pour ceux qui l'aiment encore, ou qui le respectent pour ce qu'il fut et qu'il aspire à redevenir, il est « Lorenzo », « Renzo » (I, 1), voire, avec le possessif (22), « mon Lorenzino » $(\mathrm{II}, 4)$ ou « mon Renzo » $(\mathrm{I}, 6)$. Enfin, lorsque les pairs de Lorenzo parlent de lui à des inférieurs, ils mentionnent ses titres, de manière à signifier qu'à leurs yeux comme

(22) Ce possessif indique une nuance affectueuse ou admirative forte : il est utilisé par les femmes, et, dans le registre (de la passion) politique, par Philippe Strozzi ( "Mon Brutus, mon grand Lorenzo!» $(\mathrm{V}, 2)$. 
aux yeux de tous, « le seigneur Lorenzo » est un homme respectable, dès lors qu'il rejoint le camp des ennemis du tyran ( $V, 7)$.

II n'est pas sans intérêt de remarquer que si l'on s'intéresse à la manière dont l'auteur nomme le personnage, la pièce se nomme Lorenzaccio, cependant que la liste des personnages mentionne "Lorenzo », et c'est sous ce nom qu'il prend la parole, la liste des personnages indiquant «Lorenzo de Médicis » et, entre parenthèses, "Lorenzaccio ». Cette multiplicité des dénominations traduit les hésitations du locuteur-scripteur entre un jugement positif et confraternel sur Lorenzo, et un jugement négatif sur Lorenzaccio, sans que le choix de Lorenzaccio pour le titre ne signifie que le scripteur privilégie un jugement négatif, dans la mesure où ce qui évoque une déchéance, de la pureté (= Lorenzo) au vice (= Lorenzaccio) ne nous dit rien sur la prise en charge énonciative du jugement. D'ailleurs, le fait que Lorenzo lui-même se nomme parfois "Lorenzaccio » indique qu'il se fait l'écho des jugements négatifs sur sa personne (23) : bref, l'appellatif serait dialogique, et signifierait que Lorenzo n'en a pas définitivement fini avec ses rêves de pureté d'antan (et c'est là tout son drame), quand bien même l'expérience de la " grande confrérie du vice » laisse penser que Lorenzo est devenu son personnage damné, le meurtre étant la seule échappatoire pour retrouver sa dignité perdue.

[24] Lorenzo : Suis-je un Satan? Lumière du ciel! je m'en souviens encore ; j'aurais pleuré avec la première fille que j'ai séduite, si elle ne s'était mise à rire. Quand j'ai commencé à jouer mon rôle de Brutus moderne, je marchais dans mes habits neufs de la grande confrérie du vice, comme un enfant de dix ans dans l'armure d'un géant de la fable. Je croyais que la corruption était un stigmate, et que les monstres seuls la portaient au front. J'avais commencé à dire tout haut que mes vingt années de vertu étaient un masque étouffant - ô Philippe ! (Musset, Lorenzaccio III, 3)

\subsection{Polyphonie dans les récits enchâssés monologaux : points de vue narratifs et argumentatifs}

Si le PDV se repère à travers des marques linguistiques variées, telles celles qui relèvent de la cohésion nominale (cf. Achard-Bayle 2001), comme à travers la cohésion verbale (Vogeleer 1994, Rabatel 2003g, 2003i), la cohésion logique (Ducrot 1980, Rabatel 1999, 2001b), il se manifeste aussi à travers un certain nombre de stratégies discursives, notamment le choix de telle ou telle séquence textuelle, ou de telle ou telle modalité de représentation des paroles ou des pensées ou des perceptions.

Le récit du sacrilège de Polyeucte et de Néarque détruisant les idoles par Stratonice est particulièrement significatif, car il met en scène deux PDV antagonistes, celui de Stratonice, sous la forme d'un récit, et celui des impies, sous celle d'un discours direct explicitement argumentatif. Ces PDV ne se neutralisent pas, dans la mesure où le DD est enchâssé dans un compte rendu d'événements dont l'efficacité pragmatique s'avère redoutable.

[25] Stratonice: C'est une impiété qui n'eut jamais d'exemple ;

Je ne puis y penser sans frémir à l'instant,

Et crains de faire un crime en vous la racontant.

Apprenez en deux mots leur brutale insolence.

Le prêtre avait à peine obtenu du silence,

Et devers l'orient assuré son aspect,

Qu'ils ont fait éclater leur manque de respect :

(23) Philippe : Arrête ! ne brise pas comme un roseau mon bâton de vieillesse. Je crois à tout ce que tu appelles des rêves; je crois à la vertu, à la pudeur et à la liberté.

Lorenzo : Et me voilà dans la rue, moi, Lorenzaccio ? (Musset, Lorenzaccio, III, 3) 
A chaque occasion de la cérémonie,

A l'envi l'un et l'autre étalait sa manie,

Des mystères sacrés hautement se moquait,

Et traitait de mépris les dieux qu'on invoquait.

Tout le peuple en murmure, et Félix s'en offense ;

Mais tous deux s'emportant à plus d'irrévérence:

"Quoi ? lui dit Polyeucte en élevant sa voix,

Adorez-vous des dieux ou de pierre ou de bois ? "

Ici dispensez-moi du récit des blasphèmes

Qu'ils ont vomis tous deux contre Jupiter mêmes.

L'adultère et l'inceste en étaient les plus doux.

"Oyez, dit-il ensuite, oyez peuple, oyez tous.

Le Dieu de Polyeucte et celui de Néarque

De la terre et du ciel est l'absolu monarque,

Seul être indépendant, seul maître du destin,

Seul principe éternel et souveraine fin.

C'est ce Dieu des chrétiens qu'il faut qu'on remercie

Des victoires qu'il donne à l'empereur Décie ;

Lui seul tient en sa main le succès des combats ;

Il le peut élever, il le peut mettre à bas.

Sa bonté, son pouvoir, sa justice est immense ;

C'est lui seul qui punit, lui seul qui récompense.

Vous adorez en vain des monstres impuissants »

Se jetant à ces mots sur le vin et l'encens,

Après en avoir mis les saints vases par terre,

Sans crainte de Félix, sans crainte du tonnerre,

D'une fureur pareille ils courent à l'autel!

Cieux! A-t-on vu jamais, a-t-on vu rien vu de tel?

Du plus puissant des dieux nous voyons la statue

Par une main impie à leurs pieds abattue,

Les mystères troublés, le temple profané,

La fuite et les clameurs d'un peuple mutiné,

Qui craint d'être accablé sous le courroux céleste.

(Corneille, Polyeucte III, 2, v. 822-861)

Stratonice choisit des termes péjoratifs pour évoquer Polyeucte et Néarque : " leur brutale insolence », " leur manque de respect », " Al'envil'un et l'autre étalait sa manie », " dispensez-moi du récit des blasphèmes / Qu'ils ont vomis (24) tous deux ", " d'une fureur pareille ", " tous deux s'emportant à plus d'irrévérence "; chacune de ces expressions est assortie d'un déterminant possessif de troisième personne du pluriel ou d'un adjectif qualificatif ("pareille ») ou de pronoms ( "l'un et l'autre », " tous deux ») pour bien marquer leur solidarité dans le sacrilège : telle est du moins l'image qu'en donne Stratonice. Non seulement les impies sont solidaires, mais encore seuls, face à l'immense cohorte des bons romains : le PDV de Stratonice, c'est également celui du "prêtre », de "Félix », de « tout le peuple ». Le jeu des pronoms personnels sujets est à ce titre très significatif : Stratonice commence son récit par « je », et utilise sur la fin « on » et «nous » ( Cieux ! A-t-on vu jamais, a-t-on vu rien de tel ? / Du plus puissant des dieux nous voyons la statue »), ce qui indique combien le narrateur partage les valeurs et l'indignation de sa communauté. Ainsi, son PDV se marque dans le récit des événements, dans les commentaires plus ou moins expansés de l'action, dans le choix de dénominations valorisantes ou dévalorisantes (" blasphèmes " vs " saints vases »), et par une structure antithétique qui ne cesse de construire l'opposition entre eux et nous.

(24) Les assonances en [i] montrent qu'aux oreilles de Stratonice, le contenu odieux du discours de Polyeucte et de Néarque est redoublé par un dire lui-même insupportable. 
Quant au PDV adverse, il est exprimé dans le DD rapporté par Stratonice (nous reviendrons plus loin sur la signification de cet enchâssement). On observe une même opposition entre les impies et les vrais croyants, mais, comme il était prévisible, les valeurs y sont inversées : la polarité négative est formée de l'ensemble "peuple " + «tous » + " vous » + " des dieux ou de pierre ou de bois " + " ses monstres impuissants », tandis que "Le Dieu (25) de Polyeucte et celui de Néarque ", " ce Dieu des chrétiens » représente la polarité positive. Cette opposition est toutefois subsumée par l'impératif déontique, lorsque Polyeucte invite ses compatriotes à rendre grâce à son Dieu en utilisant un pronom indéfini (" qu'il faut qu'on remercie ») : en se prévalant de cet impératif censé s'imposer à tous, Polyeucte et Néarque paraissent détruire les idoles y compris au nom des païens timorés... L'inversion des valeurs se marque également à travers l'évocation de l'omnipotence divine qui, dans le discours très militant de Polyeucte, repose sur des affirmations tranchées, répétées sur le mode de l'évidence, comme si elles n'étaient pas sujettes à discussion : les trois occurrences des "seul " puis des " lui seul » - l'adjonction de « lui » intensifiant la puissance divine - et l'amplification du rythme ternaire initial construisent une représentation du Dieu des chrétiens comme puissance indiscutable. Les rythmes sont censés incarner cette toute puissance, qu'il s'agisse du rythme ternaire, déjà mentionné (cf. encore «Sa bonté ", " son pouvoir ", " sa justice " " est immense ») ou d'un rythme binaire insistant sur l'omnipotence divine : « Il le peut élever » vs: « il le peut mettre à bas », « lui seul qui punit » vs « lui seul qui récompense », " de la terre et du ciel ». Cette toute puissance est non seulement posée, elle est aussi présupposée : ainsi d'« absolu monarque » vs monarque " absolu ».

Comme on l'a dit, le PDV de Polyeucte et Néarque, est un discours rapporté : nous en prenons connaissance comme discours enchâssé, dans un récit où c'est Stratonice qui a le dernier mot. Situation profondément inégalitaire, d'autant que Stratonice ne répond pas par un contre-discours aux arguments de ses ennemis : elle leur oppose le récit des événements, qui, indirectement, ont pour but de disqualifier la foi de Polyeucte et de Néarque. C'est pourquoi la manière dont Stratonice présente son PDV est plus forte que le PDV de ses ennemis : comme on peut toujours opposer des contre-discours aux discours (Plantin 2002 : 240-1), elle choisit pour sa part d'opposer au PDV de Polyeucte le récit incontestable des faits (une profanation) et des évidences doxiques (une impiété sans pareille). A quoi s'ajoutent des présupposés très efficaces : ainsi, la mise en relief de la " statue » " abattue » revient faire passer le jugement contenu dans l'énoncé en position thématique le "plus puissant des dieux » pour un présupposé indiscutable. De même, ce qui est en position rhématique, c'est " troublés » (« mystères »), « profané » (« temple ») : ainsi, le temple et les mystères échappent-ils à la contestation. En revanche, du point de vue de Néarque et de Polyeucte, il n'y a pas de sacrilège, puisque ces lieux, ces statues, ces vases ne sont que des signes odieux d'une stupide impiété humaine. La crainte finale du « courroux céleste " confirme bien que si Stratonice comme tous ceux qui partagent son indignation (apparemment tout le monde), craint les dieux, c'est qu'elle croit en eux, sans qu'il soit besoin d'un long discours pour cela. Ainsi ce PDV narratif témoigne-t-il d'une efficacité pragmatique plus efficace que le discours clairement argumentatif de Polyeucte.

(25) Avec une majuscule absente dans le récit de Stratonice... 


\subsection{Polyphonie des points de vue dans les monologues}

Les PDV sont donc traversés par la polyphonie, ce qui n'est pas sans poser des problèmes d'interprétation quand les repères manquent ou quand ils sont neutralisés ou brouillés. C'est ce qui se passe dans les monologues, surtout s'ils forment à eux seuls un texte théâtral, comme il s'en trouve dans la production contemporaine. II est intéressant de s'attarder sur ces textes dans lesquels le personnage cumule les rôles de je narré et de je narrant. On analysera sous l'angle du PDV et de la polyphonie L'Affaire Ducreux, de Pinget, qu'on comparera avec sa version « romanesque » antérieure, Le Libera (Bouchard et alii 2002). Ce texte repose sur une polyphonie déréglée, dans la mesure où les sources se contredisent, ce qui aboutit à une neutralisation des PDV d'autant plus problématique que les repères se brouillent entre la part du je narré et du je narrant. Et, par-dessus le tout, les PDV sont renvoyés à un univers fantasmé, dans la mesure où ce qui leur sert de support, c'est-à-dire la réalité, est sapé à la base par un « flou » référentiel généralisé.

Comme dans le récit matriciel de 1984, I'histoire (le meurtre d'un enfant) tient en quelques lignes. Mais est-ce bien un crime ? Où et quand s'est-il produit ? Qui en est l'auteur? Les réponses se contredisent, et, tout en cernant la réalité, la diluent au point qu'on hésite d'abord sur la culpabilité du personnage, ensuite sur la santé mentale du narrateur. Le texte est ainsi un tissu serré qui ressasse approximations et contradictions : tantôt la domestique a assisté au drame, tantôt elle était absente :

[26] Je n'étais même pas présente le jour du drame, chez ma sœur qui venait d'accoucher d'un troisième... Je n'ai appris ça que quinze jours après, mais j'ai tout de suite pensé à certaines personnes... motus... qui auraient bien pu être dans le coup. Pas question d'en lever la langue...

En a-t-on fait des commentaires...

Le maître d'école, un vicieux, le romano qui campe en lisière de la forêt, un détraqué, j'en donnerai mes deux mains à couper, le secrétaire de Mademoiselle, faux jeton, tous les jours guettant les enfants à la sortie de l'école, il leur offrait des bonbons... Et le sacristain même, le sacristain parfaitement, avec ses airs de bon apôtre... (Pinget, L'Affaire Ducreux, Minuit, 1995 : 14-15).

Vs

[27]

Vous souvenez-vous d'avoir vu les enfants sortir de l'école ce jour-là... Avez-vous remarqué quelque chose... Un étranger aborder le petit Ducreux... Auriez-vous remarqué que l'enfant prenait une autre direction que celle de la demeure des parents?

Oui j'ai vu le secrétaire offrir des bonbons aux enfants, je viens de le dire, selon son habitude... Ouij'ai vu le romano sortir de sa roulotte et se diriger du côté de l'école... Mais non je n'ai rien vu, j'étais chez ma sœur qui accouchait d'un troisième (Pinget, L'Affaire Ducreux, Minuit, 1995 : 16).

[28] VOIX OFF impérative et menaçante : Connaissiez-vous le petit Ducreux, un Orphelin? DOMESTIQUE : Non... Oui... Une fois rencontré sur la route de Douves. VOIX OFF : Une fois sur la route de Douves, une autre fois sur la plage? DOMESTIQUE : Sur la plage... oui... avec sa maman... (Pinget, L'Affaire Ducreux, Minuit, $1995: 23$ ).

Tantôt elle connaît la victime, tantôt non :

[29] M'interroger moi après tout ce temps, après le décès de Monsieur, qu'est-ce que ça signifie? Est-ce raisonnable ? Bien sûr que nous connaissions l'enfant, mais motus, motus... (Pinget, L'Affaire Ducreux, Minuit, 1995 : 14).

[30] Le petit Ducreux... un enfant de huit ans... Non je ne le connaissais pas... Une fois peut-être... une fois sur la route de Douves... (Pinget, L'Affaire Ducreux, Minuit, $1995: 23)$.

Tantôt le meurtre a eu lieu sur une plage, tantôt dans une forêt : 
[31] Je l'ai vu le noyé sur la plage... un enfant de huit ans... La tempête avait fait des ravages sur toute la côte... la maman ne s'en est jamais remise, séjour à l'hôpital, dépression nerveuse... et le père... il s'était mis à boire... (Pinget, L'Affaire Ducreux, Minuit, $1995: 22)$.

Vs

[32] Le petit Antoine assassiné en pleine forêt par un satyre qui est en fuite (Pinget, $L$ 'Affaire Ducreux, Minuit, 1995 : 14).

Bref, les situations comme les noms se mêlent, s'estompent, Monsieur étant sans enfant, n'ayant qu'une nièce, ou ayant un fils :

[33] Pauvre Monsieur. Il avait déjà eu bien du malheur dans sa vie... Divorcé à trente ans, un enfant à charge chez son ex, le petit Philibert, je ne l'ai connu qu'à vingt-cinq ans, il venait voir son père le dimanche.

II n'aimait que le gigot d'agneau ou les côtelettes et la purée de pommes de terre. Et la crème caramel et la mousse au chocolat... Tout juste s'il ne m'a pas fait une scène un jour que j'avais fait une tarte à la rhubarbe... (Pinget, L'Affaire Ducreux, Minuit, $1995: 11)$.

Vs

[34] N'empêche qu'il m'a écoutée et quelques jours après il en a discuté avec sa nièce qui arrivait par le train de sept heures... J'avais fait un lapin aux herbes... Elle aimait ça, gourmande comme une vieille chatte... Et de la mousse au chocolat... Tout juste si elle ne m'avait pas fait une scène un jour que j'avais fait une tarte à la rhubarbe... (Pinget, L'Affaire Ducreux, Minuit, 1995 : 21-22)

[35] Mais il [Monsieur] était fiancé à cette fille, comment s'appelait-elle... Monette... Laurette... Odette... (Pinget, L'Affaire Ducreux, Minuit, 1995 : 10).

Ailleurs, le personnage suggère que c'est le «patron » qui offrait des bonbons au petit, qui l'aimait au point de vouloir l'adopter, et que ce serait la nièce qui aurait commandité l'assassinat du petit au romanichel afin d'écarter un rival pour conserver tout l'héritage. Mais n'est-ce pas plutôt la domestique qui aurait tué le petit elle-même pour s'approprier tout l'héritage (ou à tout le moins qui y aurait pensé, et en éprouverait du remords) ? Elle répète à plusieurs reprises son statut subalterne (" oh, mes occupations à moi, celles d'une domestique ni plus ni moins ", ibid. 13 ; " moi, simple domestique », ibid. 21), oppose lourdement la richesse de Monsieur (ou du jardinier, ibid. 19) à sa misère (cf. l'évocation de sa retraite misérable pages 18-19), voire évoque les signes extérieurs de richesse en se les appropriant : "sa belle maison si confortable, oui, si confortable » (ibid. 12) ; "Mon Dieu ce que je l'ai aimée cette maison... La vue sur la rivière et les collines, la terrasse si coquette avec son mobilier blanc. [...] Et le petit bureau avec son secrétaire, troisième tiroir de gauche le testament. [...] Et la belle salle à manger » (ibid. 17-18). Cette évocation ressassée se double d'une appropriation réitérée de " notre belle vaisselle », " notre (26) maison du Midi »... Le tout en prélude à ce testament en sa faveur, qui aurait été détruit :

[36] Le notaire n'a rien voulu savoir, tout juste s'il ne m'a pas soupçonnée... Soupçonné de quoi. D'avoir renoncé à la somme que me laissait Monsieur? Toute cette clique de jemenfichistes... Les pauvres, qu'on les laisse crever... Est-ce qu'on a le droit de laisser moisir une femme de mon âge dans ces deux pièces sans chauffage ? » (Pinget, L'Affaire Ducreux, Minuit, 1995 : 18).

II n'est pas certain que cette dernière hypothèse mérite plus de crédit que les autres : tout ce qui est raconté peut s'interpréter aussi bien comme un aveu du je narré que comme un regret de n'avoir pas osé passer à l'acte, voire comme une

(26) Cf. encore "Quant aux parents, ils ferait beau voir qu'ils nous mêlent à cette affaire... " (ibid. 15). 
simple projection gratuite, un délire sans culpabilité du je narrant. Cette succession kaléidoscopique de PDV contradictoires sans aucune hiérarchisation, voire sans fondement référentiel fiable et stable, qui se clôt sur le vertige de la page blanche, renvoie en définitive au théâtre intérieur du personnage... avec un refus absolu de transcendance : exit le libera, il ne reste qu'un sombre fait divers, une rumeur, du bruit et de la fureur.

L'exemple du Libera illustre la dimension polyphonique du PDV ainsi que sa dimension interactionnelle, y compris en contexte monologal. Nous voudrions pour conclure insister sur cette proximité, qui est au cœur du texte théâtral, dès l'origine.

\title{
3. LA FONCTION ANTHROPOLOGIQUE DU PDV AU THÉÂTRE, AGENT DOUBLE NARRATIVO-ARGUMENTATIF, MACHINE À RESSORT DRAMATIQUE ET OUTIL DE LA PENSÉE RATIONNELLE
}

On a pu constater en 2. combien le texte théâtral intrique PDV narratif et argumentatif, effets pragmatiques directs ou indirects. Le fait que le PDV soit une sorte d'agent double (Rabatel 2000), argumentativo-narratif, n'est pas sans évoquer d'une façon quasi consubstantielle la nature ambivalente du théâtre. Ce caractère hybride du texte théâtral fait écho aux travaux récents qui mettent en relation, d'un point de vue anthropologique, le rôle de la tragédie grecque antique dans l'émergence de la rhétorique. Nous prolongerons cette hypothèse, tout en la recontextualisant dans le cadre d'une histoire culturelle dont il faut souhaiter le développement. Danblon 2003 montre que la tragédie modifie la valeur anthropologique intrinsèque des récits (cf. I'hypothèse génétique et cognitive de Victorri 2002) en permettant à la rationalité humaine de se déployer, par la construction de points de vue distincts, qui ont pour fonction de complexifier l'appréhension du réel et, partant, d'aider à la régulation des problèmes de la collectivité :

\begin{abstract}
Chez les pré-hominidés, la fonction narrative aurait pris le relais lorsque les contraintes biologiques se sont estompées. Un ancien rapportait au sein du groupe le souvenir d'un événement traumatisant à l'origine d'une crise sociale mémorable afin de permettre à la communauté de les éviter. Les premières sociétés orales auraient trouvé, dans de telles habitudes discursives, une manière de jurisprudence qui puisait sa topique propre dans la mémoire orale des anciens. Les grandes histoires mythiques ont peut-être émergé à partir de ces premières habitudes narratives. Or, le processus narratif se complexifie dans la tragédie : la description des faits prend du relief, en même temps qu'émerge la notion de point de vue - celui du chœur, celui des personnages. Le rapport d'événements commence à acquérir une profondeur épistémique qu'il ne possédait pas auparavant. Un tel relief épistémique, qui dévoile les notions d'intention, de culpabilité, de responsabilité individuelle, de qualification juridique, reste bien sûr totalement étranger au mythe. Les notions émergentes seront ensuite théorisées dans le genre judiciaire de la rhétorique par le biais du questionnement, propre au travail de la critique (Danblon 2003).
\end{abstract}

Cette analyse met en relief le rôle charnière de la notion de PDV, qui relève d'une part de l'univers mythique et narratif dont elle procède et qu'elle contribue à complexifier, d'autre part de l'univers discursif et rationnel qu'elle annonce, et qu'elle construit. A vrai dire, Danblon ne dit pas explicitement que le PDV joue un rôle d'intermédiaire entre mythe et argumentation, elle réserve cette place à la tragédie, dans laquelle se joue « une pensée juridique en train de s'élaborer », autour de deux conceptions de la faute, avec « une ambiguïté, une hésitation non résolue entre la vieille faute homérique et la faute juridique historiquement en cours d'élaboration (qui utilise quant à elle les notions d'intention et de responsabilité individuelle) » (Danblon 2003) : 
Dans la pensée mythique, nature et politique se confondent ; les lois ne doivent pas être fondées, elles ont le même statut que les faits : tout est évident. Le monde est clos et la critique est absente. Le mythe est notoirement marqué par la coexistence de termes contradictoires et cette situation est sans doute en partie liée à l'usage peu réflexif qui est fait du langage dans un mode de pensée principalement oral. Dans ces sociétés, la narration assume une fonction de transmission d'information et de prise de décision, sans que la dimension fictionnelle soit pertinente à ce stade. La narration des sociétés orales remplit donc les fonctions qui seront prises en charge, postérieurement, par les genres délibératif (prendre des décisions) et judiciaire (rendre des jugements) de la rhétorique. La fonction narrative joue donc au départ un rôle capital dans la vie politique et sociale où elle assumait sans doute une telle fonction informative et directive, aussi longtemps qu'aucune polysémie n'était conscientisée : le récit collait aux faits, aucune interprétation ne venait rendre la parole potentiellement ambiguë et donc sujette à diverses interprétations. Mais la fonction politique de la narration est rendue problématique dans la tragédie ; par l'introduction d'une dissociation inédite entre mythe et logos, entre parole collective et parole individuelle, émerge la notion de fiction. Dans la poésie tragique, les histoires traditionnelles sont traitées par un filtrage de points de vue qui leur donne un nouveau relief épistémique. Ainsi, chez Sophocle, le meurtre de Laïos est présenté tantôt comme une histoire, tantôt comme un événement rapporté par un témoin. Pour Gould (2001), la tragédie utilise simultanément les diverses formes discursives que sont le mythe, la narration, la gnome et l'argumentation; il y aurait dans la tragédie simultanément une mémoire racontée et une explication raisonnée. Mais par la dimension gnomique couplée à l'apparition des points de vue, l'argumentation se profile derrière la narration : simultanément petite histoire exemplaire à caractère proverbial et topique à caractère pré-inférentiel. La tragédie témoigne d'une importante mutation cognitive et politique : la loi, le droit et le langage s'affirment désormais comme des pratiques humaines, les hommes prennent conscience de la réalité du monde qu'ils ont inventé : celui de la réalité sociale. La tragédie est le lieu où cette prise de conscience se produit et s'exprime, sans être encore résolue (Danblon 2003).

II nous semble à peine forcer l'analyse de Danblon en insistant sur le fait que le PDV est un des moteurs essentiels de la pensée rationnelle en cours de structuration. Si l'hypothèse est pertinente (27), il est probable que le processus de construction/transformation de la pensée rationnelle ne s'achève pas avec la tragédie, mais qu'il se poursuit au contraire avec la comédie. Cette extension n'est pas infondée, si l'on considère que ces deux genres théâtraux ont en commun de représenter (en un mot) des conflits, de permettre aux opinions personnelles de se développer (de se construire, comme on l'a dit plus haut) à travers les interactions elles-mêmes, et surtout à travers des mécanismes de re-présentation (28) caractéristiques de la comédie.

En effet, avec la comédie, le PDV se développe en se portant sur des domaines plus triviaux, et en se rapportant à des personnages ordinaires. Le personnage comique s'affronte à des problèmes humains ordinaires, éloignés de leur naturalisation dans le mythe ou de leur sacralisation dans la tragédie (cf. Vernant, Girard). Si, dans la tragédie, le conflit des valeurs est intériorisé, le héros tragique étant le lieu même où se combattent des valeurs antithétiques, dans la comédie en revanche, le conflit s'extériorise : alors que le spectateur/lecteur souffre avec le héros tragique, dans la

(27) Cette hypothèse demanderait à être vérifiée dans le cadre d'une histoire anthropologico-culturelle (et donc littéraire) qui s'intéresserait aux genres et aux œuvres à partir de la notion de PDV. Ce travail reste à faire, avis aux amateurs!

(28) La re-présentation renvoie à une présentification par le biais du comme-si et souligne la valeur cognitive de la mimèsis (Aristote), qu'il faut distinguer de la mimèsis comme feintise (Platon) : cf. Schaeffer 1999, chapitre 1. La conception aristotélicienne de la mimèsis comme connaissance des faits nécessaires, vraisemblables ou possibles s'accommode assez bien de l'hypothèse de Victorri. Évidemment, cette hypothèse est différente de celle de Nietzsche, qui, dans La naissance de la tragédie, interprète l'avènement de la représentation mimétique comme le signe d'un abâtardissement de la pensée religieuse en une représentation magique, mais notre propos n'est pas de confronter les hypothèses en présence. 
comédie, en revanche, il a plutôt tendance à rire du héros comique, d'autant plus sûrement que la représentation grossit les faits comme leurs effets. La comédie antique, notamment chez Aristophane, met en scène des personnages et des situations politiques appréhendés sur un mode ludique et grossi (29) favorisant la vis comica et la distanciation critique : Lysistrata traite de la question de la guerre et de la paix Les Guêpes du (dys)fonctionnement de la justice, Les Cavaliers des errements du peuple qui se donne à des démagogues sans scrupules, à travers les joutes du Paphlagonien, du Charcutier devant Démos, Les Oiseaux de la question du communisme des biens et des femmes, etc. La comédie peut ainsi être analysée comme un intermédiaire de plus, s'ajoutant à la tragédie, dans l'émergence de la rationalité sous sa forme rhétorique (30), parce qu'elle repose sur le jeu, sur de multiples structures en comme-si, dont la dimension cognitive est désormais bien connue (Danblon 2002). Ces structures mimétiques dont la dimension cognitive est redoublée par les phénomènes de mise à distance reposent essentiellement sur l'utilisation à des fins dramatiques des topoi, des jeux de rôle, des stratagèmes et des épreuves, qui culminent dans la mise en abyme du comme-si à travers le théâtre dans le théâtre. Plutôt que de continuer à prendre des exemples dans l'antiquité, nous nous référerons à présent à des exemples classiques, susceptibles de faire sens pour un public scolaire. Le fait que ces exemples renvoient au XVII ${ }^{\mathrm{e}}$ ou au XVIII ${ }^{\mathrm{e}}$ siècles, plutôt qu'à l'antiquité grecque, n'enlève rien à notre hypothèse : ces valeurs sémiologiques de la comédie perdurent au-delà des variations génériques, et sont susceptibles de se cumuler (Danblon 2002 : 233-235) avec des valeurs spécifiques, selon les époques, comme on le verra rapidement pour le théâtre de Marivaux.

\subsection{Topoi}

La comédie participe du développement de la pensée rationnelle en permettant au PDV de jouer avec les topoi pragmatiques ou rhétoriques, ou de favoriser leur mise à distance. En [37], la description de Hyacinte repose sur le topos du coup de foudre, et sur le stéréotype de la jeune fille simple, pauvre, mais aimable par ses seuls charmes " naturels "; en [38] et [39], les valets procèdent à des inférences distanciées à l'égard du récit très codé d'une situation dont ils sont les observateurs ironiques:

[37] Octave : Une autre aurait paru effroyable en l'état où elle était ; car elle n'avait pour habillement qu'une méchante petite jupe avec des brassières de nuit qui étaient de simple futaine, et sa coiffure était une cornette jaune, retroussée au haut de sa tête, qui laissait tomber en désordre ses cheveux sur ses épaules; et cependant, faite comme cela, elle brillait de mille attraits, et ce n'était qu'agrément et que charmes que toute sa personne (Molière, Les Fourberies de Scapin, I, 2).

Ces stéréotypes, dont le maître (l'amoureux) ne se rend pas compte, et qu'il rapporte avec délice (31), sont néanmoins immédiatement repérés par les valets (non amoureux), qui exhibent les inférences qui s'ensuivent :

[38] "Je sens venir les choses", «Oh, je n'en doute point; et sans l'avoir vue, je vois bien qu'elle était tout à fait charmante », « je vois bien que ce bon naturel-là vous la fit aimer » (Molière, Les Fourberies de Scapin, I, 2).

(29) Ainsi de la comédie amoureuse que Myrrhine joue à son époux Cinésias, dans Lysistrata, où l'on voit que Myrrhine s'amuse des topoi du désir masculin...

(30) Sur un plan phylogénétique, mais aussi ontogénétique : cf. la littérature psychologique consacrée à l'importance du jeu dans la construction de la pensée rationnelle chez le jeune enfant, chez Piaget, Winnicott, François 1993, etc.

(31) De même qu'il est souvent observé que lorsque le DIL est repéré, il induit un changement de ton et de rythme chez les lecteurs, le repérage de fragments descriptifs focalisés par un personnage entraîne de semblables effets à l'oral. 
De la même manière Silvestre, dans son récit dialogique (racontant à Scapin la fin de l'aventure d'Octave), insiste sur des topoi, ce qui lui permet de raconter (et de juger) de façon distanciée le comportement amoureux prévisible de son maître :

[39] Silvestre, à Octave : Si vous n'abrégez ce récit, nous en voilà pour jusqu'à demain. Laissez-le moi finir en deux mots. (A Scapin) Son cœur prend feu dès ce moment. II ne saurait plus vivre qu'il n'aille consoler son aimable affligée. Ses fréquentes visites sont rejetées de la servante, devenue la gouvernante par le trépas de la mère : voilà mon homme au désespoir. II presse, supplie, conjure : point d'affaire. On lui dit que la jeune fille, quoique sans bien et sans appui, est de famille honnête et qu'à moins que de l'épouser, on ne peut souffrir ses poursuites ; voilà son amour augmenté par les difficultés. II consulte dans sa tête, agite, raisonne, balance, prend sa résolution ; le voilà marié avec elle depuis trois jours (Molière, Les Fourberies de Scapin, I , 2).

L'ironie de ce récit réside en ce que les actions sont rapportées sous la forme d'un récit de paroles, avec DIL ( "II ne saurait plus vivre qu'il n'aille consoler son aimable affligée »), discours narrativisé, pour les paroles (« il presse, supplie, conjure »), et psycho-récit, pour les pensées ( II consulte dans sa tête, agite, raisonne, balance, prend sa résolution »), rendant les personnages comme transparents au yeux du narrateur « omniscient » qu'est Silvestre. Son récit est condensé car la situation est jugée terriblement banale, ce qui lui permet de se moquer d'un maître qui est la dupe consentante de la comédie amoureuse. La succession des verbes produit un effet d'accélération qui donne à Octave l'allure d'un pantin agi par la situation (et peut-être par la belle, comme par la gouvernante) ; c'est d'ailleurs ce que semble indiquer la didascalie qui place Octave en position d'interlocuteur délocuté ( $c f$. « mon homme ») : il est celui dont on parle comme il a été celui qu'on a manipulé comme une marionnette.

\subsection{Jeux de rôles}

Ces mises à distance se combinent avec la représentation des topoi sous la forme de jeux de rôles, permettant au PDV d'explorer la réalité, de jouer avec des mécanismes de construction des identités sociales, de ressorts psychologiques, tout en participant à la construction des effets dramatiques :

[40] Scapin : Çà, essayons un peu pour vous accoutumer. Répétons un peu votre rôle, et voyons si vous ferez bien. Allons. La mine résolue, la tête haute, les regards assurés.

Octave : Comme cela?

Scapin : Encore un peu davantage.

Octave : Ainsi?

Scapin : Bon! Imaginez-vous que je suis votre père qui arrive, et répondez-moi fermement, comme si c'était à lui-même. "Comment ! pendard, vaurien, infâme, fils indigne d'un père comme moi, oses-tu bien paraître devant mes yeux après tes déportements, après le lâche tour que tu m'as joué pendant mon absence ? [...] Répondsmoi, coquin, réponds-moi ! Voyons un peu tes belles raisons ! » Oh ! que diable ! vous demeurez interdit? (Molière, Les Fourberies de Scapin, I , 3).

[41] Scapin : Je cherche seulement dans ma tête un homme qui nous soit affidé, pour jouer un personnage dont j'ai besoin. Attends. Tiens-toi un peu. Enfonce ton bonnet en méchant garçon. Campe-toi sur un pied. Mets la main au côté. Fais les yeux furibonds. Marche un peu en roi de théâtre (Molière, Les Fourberies de Scapin, I , 5).

Ces mécanismes, ici en congruence avec les canons de la commedia dell'arte peuvent renvoyer à d'autres époques, à d'autres esthétiques, ou éthiques, en tout cas recèlent, en sus de leur valeur dramatique, une dimension cognitive importante, car ces jeux permettent au moi de s'éprouver en (se) jouant la comédie (de l'amour, du pouvoir, etc.) : d'où le rôle de la notion d'épreuve. 


\subsection{L'épreuve mise en scène}

L'épreuve jouée correspond à une des formes dramatiques majeures de la mise en scène du comme-si : le héros tragique ne joue pas, il cherche vainement à perdurer dans son être ; en revanche, le héros comique surmonte des conflits embrouillés puis résolus par des stratagèmes (32), des tests. La dimension cognitive de ces épreuves n'existe nulle part ailleurs mieux que dans le théâtre de Marivaux, où la machine à comique croise le développement des sciences et de l'esprit d'observation dans les sciences naturelles notamment (33), qui jouent un rôle structurant dans l'épistémè du XVIII ${ }^{e}$ siècle. Ainsi du Prince de La Dispute ([42]), ou de Lucidor, dans L'Epreuve ([43]) :

[42] Le prince : pour bien savoir si la première inconstance ou la première infidélité est venue d'un homme, comme vous le prétendez, et moi aussi, il faudrait avoir assisté au commencement du monde et de la société.

Hermiane : Sans doute, mais nous n'y étions pas.

Le prince: Nous allons y être ; oui, les hommes et les femmes de ce temps-là, le monde et ses premières amours vont reparaître à nos yeux tels qu'ils étaient, ou du moins tels qu'ils ont dû être ; ce ne seront peut-être pas les mêmes aventures, mais ce seront les mêmes caractères ; vous allez voir le même état de cœur, des âmes tout aussi neuves que les premières, encore plus neuves s'il est possible [...] Voici le fait : il y a dix-huit ou dix-neuf ans que la dispute d'aujourd'hui s'éleva à la cour de mon père, s'échauffa beaucoup et dura très longtemps. Mon père, naturellement assez philosophe, et qui n'était pas de votre sentiment, résolut de savoir à quoi s'en tenir par une épreuve qui ne laissât rien à désirer. Quatre enfants au berceau, deux de votre sexe et deux du nôtre, furent portés dans la forêt où il avait fait bâtir cette maison exprès pour eux, où chacun d'entre eux fut logé à part, et où actuellement même il occupe un terrain dont il n'est jamais sorti, de sorte qu'ils ne se sont jamais vus [...] On va donc pour la première fois leur laisser la liberté de sortir de leur enceinte, et de se connaître ; on leur a appris la langue que nous parlons ; on peut regarder le commerce qu'ils vont avoir ensemble comme le premier âge du monde ; les premières amours vont recommencer, nous verrons ce qui en arrivera (Marivaux, La dispute, Garnier-Flammarion, 1991: 152).

[43] Lucidor : Tout sûr que je suis de son cœur, je veux savoir à quoi je le dois ; et si c'est l'homme riche qu'on aime, c'est ce que j'éclaircirai par l'épreuve où je vais la mettre ; il m'est encore permis de n'appeler qu'amitié tout ce qui est entre nous deux, et c'est de quoi je vais profiter (Marivaux, L'Epreuve, Garnier-Flammarion, 1991: 41).

\subsection{Mise en abyme du comme-si}

La comédie participe également au développement de la pensée rationnelle en favorisant maintes formes de mise en abyme du comme-si qui offrent des occasions privilégiées pour complexifier les PDV. Ce comme-si alimente toute une palette de jeux, de secrets, de doubles (34), qui ont une fonction cognitive importante, redoublée par leur re-présentation dédoublée. Le comme-si fonctionne au deuxième degré, comme machine à théâtre et alimente le développement de la pensée rationnelle à travers la construction d'univers contrefactuels, hypothétiques, de nature à vérifier, alimenter des inférences hypothético-déductives, etc. :

(32) Cf. Scapin, maître ès « inventions », ès " machines ", ès «fabriques de ces gentillesses d'esprit, de ces galanteries ingénieuses à qui le vulgaire ignorant donne le nom de fourberies » (Molière, Les fourberies de Scapin, I,1) ou encore expert en " honnête petit stratagème » (ibid. I, 2).

(33) Cf. Roger 1963, cité in Goldzink 1991: 214.

(34) Dans sa présentation des Acteurs de bonne foi : Goldzink 1991: 216 cite comme figures du double « le théâtre et la vie, les maîtres et les valets, les hommes et les femmes, les jeunes et les vieux, les riches et les moins riches, les manipulateurs et les manipulés ». 
[44] Merlin : J'oublie encore de vous dire une finesse de ma pièce, c'est que Colette qui doit faire mon amoureuse, et moi qui dois faire son amant, nous sommes convenus tous deux de voir un peu la mine que feront Lisette et Blaise, à toutes les tendresses naïves que nous prétendrons nous dire ; et le tout, pour éprouver sils n'en seront pas un peu alarmés et jaloux, car vous savez que Blaise doit épouser Colette, et l'amour nous destine, Lisette et moi, l'un à l'autre (Marivaux, Les acteurs de bonne foi, Garnier-Flammarion, $1991: 228$ ).

Or Blaise résume on ne peut mieux l'ambiguïté d'un jeu malgré tout sérieux, car on ne fait jamais impunément « semblant de faire semblant » :

[45] Blaise : Et au par-dessus on se raille de ma personne dans ce peste de jeu-là; noute maîtresse, Colette y fait semblant d'avoir le cœur tendre pour Monsieur Merlin, Monsieur Merlin de li céder le sien, et maugré la comédie tout ça est vrai, noute maîtresse ; car ils font semblant de faire semblant (Marivaux, Les acteurs de bonne foi, Garnier-Flammarion, $1991: 263$ ).

Ainsi le texte de théâtre fait une large place à la notion générique de PDV. Par son aptitude à renvoyer à des PDV exprimés par des locuteurs ou des énonciateurs, le PDV est un instrument incomparable pour rendre compte de la dimension polyphonique du langage, en contexte interactionnel. La nature argumentativonarrative du PDV fait particulièrement sens à propos du texte de théâtre, parce qu'il repose sur une mise en espace des conflits qui en favorise l'expression, voire l'expressivité, d'où l'intérêt d'une articulation du PDV avec l'ethos. Le PDV manifeste grâce à ses mécanismes énonciatifs et à sa dimension pragmatique une dimension argumentative directe ou indirecte, à même de motiver les interactions entre personnages, ou d'enrichir les mécanismes herméneutiques, notamment par l'analyse des postures de co-énonciation, de sous-énonciation ou de sur-énonciation. 


\section{Références bibliographiques}

ACHARD-BAYLE, Guy (2001) : Grammaire des métamorphoses. Bruxelles : Duculot.

AdAm, Jean-Michel et PetiTJeAn, André (1989) : Le texte descriptif. Paris : Nathan.

Amossy, Ruth (Dir) (1999) : Images de soi dans le discours. Paris, Lausanne : Delachaux et Niestlé.

- (2000) : L'argumentation dans le discours. Paris : Nathan.

BAL, Mieke (1977) : Narratologie. Paris : Klincksieck.

BANFIELD, Ann (1995): Phrases sans parole. Paris : Le Seuil.

Bouchard, Robert, Martinie, Bruno, Rabatel, Alain (2002) : " "Déclencher le mécanisme"... de la construction / déconstruction du texte romanesque ", Les modèles du discours au défi d'un "dialogue romanesque » : l'incipit du roman de R. Pinget, Le Libera, Roulet, E. et Burger, M. (éds), 153-211. Nancy : Presses Universitaires de Nancy.

Charaudeau, Patrick et Maingueneau, Dominique (2002) : Dictionnaire d'analyse du discours. Paris : Le Seuil.

Charolles, Michel (1988) : "Les plans d'organisation textuelle », Pratiques 57, 3-13.

COHN, Doritt (1980) : La transparence intérieure. Paris : Le Seuil.

Comte-SPOnVILle, André (1988) : Le mythe d'Icare. Traité du désespoir et de la béatitude, tome 1. Paris: Presses Universitaires de France.

Damasio, Antonio (1994) : Descartes' Error. Emotion, Reason, and the Human Brain. Grosset/Putnam Books [1999: L'erreur de Descartes. Paris : Odile Jacob]

- (1999): The Feeling of What Happens. Body and Emotions en the Making of Consciousness. New-York : Harcourt Brace \& Compagny. [1999 : Le sentiment même de soi. Corps, émotions, conscience. Paris : Odile Jacob]

DANBLON, Emmanuelle (2002) : Rhétorique et rationalité. Essai sur l'émergence de la critique et de la persuasion. Bruxelles : Éditions de l'Université de Bruxelles.

- (2003) : « Du tragique au rhétorique », in La tragédie et sa rhétorique, Hoggaert, C. (éd), Paris : Presses Universitaires de France.

Ducrot, Oswald (1980) : «Analyses pragmatiques », Communications 32, 11-60.

- (1984) : Le Dire et le dit. Paris : Minuit.

Genette, Gérard (1972) : Figures III. Paris : Le Seuil.

- (1983) : Nouveau discours sur le récit. Paris : Le Seuil.

ISACHAROFF, M. (1985) : Le spectacle du discours. Paris : Corti.

JEANNERET, Thérèse (1999) : La coénonciation en français. Berne : Peter Lang.

JouvE, Vincent (1992) : L'effet personnage dans le roman. Paris : Presses Universitaires de France.

- (1993) : La lecture. Paris : Hachette.

Kerbrat-Orecchioni, Catherine (1984) : "Pour une approche pragmatique du dialogue de théâtre », Pratiques 41, 46-62.

LINTVELT, Jaap (1981) : Essai de typologie narrative. Paris Corti.

MaINGUENEAU, Dominique (1984) : Genèses du discours. Bruxelles : Mardaga. - (1987) : Nouvelles tendances en analyse du discours. Paris : Hachette.

- (1990) : Pragmatique pour le texte littéraire. Paris : Bordas.

MARNETTE, Sophie (2002) : "Étudier les pensées rapportées en français : mission impossible ? », Faits de Langues 19, 211- 220.

MEIzoz, Jérôme (2003) : « ethos, champ et facture des œuvres : recherches sur la "posture" », Pratiques 117-118, 241-250.

Nonnon, Elisabeth (1999): "La notion de point de vue dans le discours », Pratiques 100.

Petitjean, André (2004) : "Lire le texte théâtral », in La lecture littéraire, V. Jouve éd., Actes du colloque "L'expérience littéraire », Université de Reims, à par.

Plantin, Christian (2002) : "Analyse et critique du discours argumentatif », in Après Perelman. Quelles politiques pour les nouvelles rhétoriques ?, Koren, R. et Amossy, R. (éds), pp. 229-263. Paris : L'Harmattan.

RABATEL, Alain (1997a) : "L'introuvable focalisation externe », Littérature 107, 88-113. — (1997b) : Une histoire du point de vue. Paris, Metz : Klincksieck/CELTED, Université de Metz.

- (1998) : La construction textuelle du point de vue. Lausanne, Paris : Delachaux et Niestlé.

- (1999) : «Mais dans les énoncés narratifs : un embrayeur du point de vue et un organisateur textuel », Le Français Moderne LXVII, 1, 49-60. Paris.

- (2000) : «Un, deux, trois points de vue ? Pour une approche unifiante des points de 
vue narratifs et discursif », La Lecture Littéraire 4, 195-254. Paris : Klincksieck/Université de Reims.

- (2001a) : « Fondus enchaînés énonciatifs. Scénographie énonciative et points de vue », Poétique 126, 151-173.

- (2001b) : «La valeur délibérative des connecteurs et marqueurs temporels mais, cependant, maintenant, alors, et dans l'embrayage du point de vue. Propositions en faveur d'un continuum argumentativo-temporel », Romanische Forschungen 113, 2, 153-170. Frankfurt am Main : Vittorio Klostermann.

- (2001c) : "Les représentations de la parole intérieure. Monologue intérieur, discours direct et indirect libres, point de vue », Langue Française 132, 72-95.

- (2002) : « Le sous-énonciateur dans les montages citationnels : hétérogénéités énonciatives et déficits épistémiques », Enjeux 54, 52-66.

- (2003a) : "Sur-énonciateurs et construction dissensuelle des savoirs », Didactiques de l'oral, Maurer, B. (éd). CRDP de Basse-Normandie, 89-100.

- (2003b) : «Les verbes de perception en contexte d'effacement énonciatif : du point de vue représenté aux discours représentés ॥, Travaux de linguistique 46-1, 49-88.

- (2003c) : "Déséquilibres interactionnels et cognitifs, postures énonciatives et coconstruction des savoirs : co-énonciateurs, sur-énonciateurs et archi-énonciateurs ", in Interactions orales en contexte didactique. Mieux (se) comprendre pour mieux (se) parler et pour mieux ( $\left.s^{\prime}\right)$ apprendre, Rabatel, A. (éd). Presses Universitaires de Lyon (sous presse).

- (2003d) : "La narrativisation d'un texte argumentatif : mode de résolution des conflits et mode d'argumentation propositive indirecte ", in Les processus de la rédaction collaborative. Mondada, L. et Bouchard, R. (éds). Paris : L'Harmattan.

- (2003e) : « L'effacement énonciatif et ses effets pragmatiques de sous- et de sur-énonciation ", in Formes et stratégies du discours rapporté : approche linguistique et littéraires des genres de discours, Lopez Muñoz, J.M., Marnette, S. et Rosier, L. (éds), Estudios de Lengua y Literatura francesas 14, 27-48. Université de Cadix.

- (2003f) : "Les formes d'expression de la pré-réflexivité dans le discours indirect libre et dans les points de vue représentés ou embryonnaires ", in Le discours indirect libre, Mathis, G. De Mattia, M. et Pégon, C. (éds). Bulletin de la Société de Stylistique anglaise 24 .

- (2003g) : « Analyse aspectuo-temporelle de l'IMP dans les suites PS + IMP sans lien thématique ", in Le Texte et les discours. Catégories pour l'analyse, J.-M. Adam, M. Ali Bouacha et J.B. Grize (éds). Dijon : Éditions universitaires de Dijon, 83-92.

- (2003h) : "Re-présentation des formes pré-réflexives dans les comptes rendus de perception, de parole et de pensée. La question du mimétisme dans les discours représentés ", in Lingvistisk og litteraer polyfoni. (La polyphonie textuelle. Polyphonie - linguistique et littéraire) 7, 1-31. Roskilde : Université de Roskilde, Danemark.

- (2003i) : « Une lecture énonciative des hypothèses aspectuo-temporelles et commentatives dans les suites PS + IMP : point de vue du locuteur ou de l'énonciateur ? ". Journal of French Language Studies, 13-1, 1-17.

- (2004) : Argumenter en racontant. Bruxelles : Duculot (à paraître).

RYNGAERT, Jean-Pierre $(1991,1999)$ : Introduction à l'analyse du théâtre. Paris : Nathan.

SCHAEFFER, Jean-Marie (1999): Pourquoi la fiction ? Paris : Seuil.

UBERSFELD, Anne (1996): Lire le théâtre, 3. Paris : Belin.

VICTORRI, Bernard (2002) : « Homo narrans : le rôle de la narration dans l'émergence du langage ", Langages 146, 112-125.

Vitoux, Pierre (1982) : « Le jeu de la focalisation », Poétique 51, 359-368.

VION, Robert " "Effacement énonciatif" et stratégies discursives », De la syntaxe à la narratologie énonciative, De Mattia, M. et Joly, A (éds), 331-354. Gap : Ophrys.

Vogeleer, Svetlana (1994): "Le point de vue et les valeurs des temps verbaux », Travaux de Linguistique 29, 39-58.

WILMET, Marc $(1997,1998)$ : Grammaire critique du français. Paris : Hachette. 


\section{Revue Siècle 21, Littérature \& Société}

Née fin 2002, la revue Siècle 21 (semestrielle, 160 pages) " se fixe comme but d'aborder les grandes questions de société et les littératures mondiales à travers la vision des écrivains de tous les pays et de tous les temps " (éditorial du $n^{\circ} 1$ ).

Chaque numéro comporte un gros dossier sur une littérature particulière (textes introductifs ou théoriques et surtout, textes littéraires), deux dossiers thématiques, des chroniques, et des dessins inédits du même artiste qui en assurent l'unité graphique.

Sont privilégiés les textes inédits ou les traductions inédites en français.

\section{Au sommaire du dernier numéro ( $n^{\circ} 3$, automne 2003) :}

Imaginaire de Saint-Pétersbourg : Hélène Henry, Bitov, Krivouline, Schwarz, lasnov, Youriev, Berg, Skidan, Stratanovski, Martynova, Barskova, Oulanovskaia, Kononov, Zavialo, Sekatski

Scatologie, pornographie, érotisme : Jean Guiloineau, Rabelais, Rimbaud, Verlaine, Ronsard, Tabarin, Claude le Petit, Théophile Gautier, Flaubert, Restif de la Bretonne, Sade, Gervaise de la Touche, Jean l'Ange, Michel Millot

Le jeu : Baudelaire, Jacques Lacarrière, Salman Rushdie, François Caradec, Carine Hahn, Abel Pohulanik, Ozren Kebo, Mellin de Saint-Gelais, Marie Etienne, le Mahâbhârata, Mircea Cartarescu.

Collages de Patrick Renisio.

\section{Au sommaire du $n^{\circ} 2$ :}

La littérature africaine francophone L'étranger, l'autre

La mesure du temps

\section{Au sommaire du $n^{\circ} 1$ :}

La littérature indienne

Hugo vu d'ailleurs

La guerre et la paix

\section{Prochains numéros:}

- $n^{\circ}$ 4. La littérature birmane. La Ballade de la geôle de Reading d'Oscar Wilde (nouvelle traduction).

Viv $\circledast$ e la traduction.

$-n^{\circ} 5$. La littérature mexicaine. La mort de la mère. Les écrivains et le cinéma

$-n^{\circ} 6$. La littérature portugaise. Marges et lisières.

\section{Comité de rédaction}

Jean Guiloineau (directeur de la publication), Sophie Képès, Catherine Pierre, Jérôme Vérain, Tirthankar Chanda, Marie-Claudette Kirpalani, Catherine Bricout, Monica Valby, Josette Rasle, Jean-Marie Chevrier

\section{Diffusion}

— En librairie : prix du numéro : 17 euros ( $\mathrm{N}^{\circ} 1: 20$ euros)

- Abonnements :

Numéros 2 et 3 ou 3 et 4 : France, 30 euros ; Dom-Tom et étranger, 35 euros. Abonnement de soutien (deux numéros) : 70 euros.

Chèque à adresser à Siècle 21, 41 rue Bobillot, 75013 Paris

\section{Renseignements}

Jean Guiloineau : jeanguiloineau@wanadoo.fr.

Marie-Claudette Kirpalani : mckirpa@wanadoo.fr 